\title{
Flowfield Evolution vs. Lift Coefficient History for Rapidly- Pitching Low Aspect Ratio Plates
}

\author{
Kenneth Granlund ${ }^{1}$ and Michael $\mathrm{OL}^{2}$ \\ U.S. Air Force Research Laboratory, Wright-Patterson Air Force Base, OH 45433, U.S.A. \\ Luis Bernal ${ }^{3}$ \\ University of Michigan, Ann Arbor, MI 48109-2140
}

\begin{abstract}
We extend recent results on 2D (wall to wall) flat plates executing various pitching motions, to rectangular and elliptical (Zimmerman planform) plates of aspect ratio 2, at reduced frequency up to 2.0. Motions include, broadly, two kinds. The first is a smoothed linear pitch from $0^{\circ}$ to $90^{\circ}$ incidence angle, with constant freestream and with relative free-stream linearly decelerating to zero towards motion completion, where the operative question is how the motion dynamics mediates stall. The second is a linear pitch ramp-hold-return maneuver, where one is interested not only in the dynamic stall response, but also return from stall. Surveying a range of pivot point locations and pitch rates, we compare the development of leading edge vortices, tip vortices and trailing edge vortices, with trends in lift coefficient and drag coefficient history. For highfrequency motions, the lift peak no longer closely correlates with the maximum strength of the leading edge vortex, or with its shedding. Instead, vortex formation and shedding can lag both the motion and the lift peak. Tip vortices evolve more slowly than leading edge vortices, in the sense that a tip vortex remains coherent and nearly attached to the wingtip until well past leading edge vortex saturation and shedding. Qualitatively the low aspect ratio plates behave as does the 2D plate; for example, taking the pitch pivot point further aft, results in both lower lift and drag peaks; and taking a very high reduced frequency of pitch, results in initial lift transients akin to those with outright zero free stream flow. Broadly, the lift history of the low aspect ratio plates has lower slope than for the 2D plate, but not with the same proportionality that holds for the steady case. However, classical scaling relations for lift as a function of reduced frequency hold nearly as well for the low aspect ratio plates, as they do for the $2 \mathrm{D}$ plate.
\end{abstract}

\section{Introduction}

The problem of transient pitch of a 2D airfoil or plate has a long history in unsteady aerodynamics. Study of high-frequency effects is useful from the viewpoint of applications such as maneuvering aircraft, gust response and flapping-wing aerodynamics. High dimensionless rates are much easier to attain in liquid facilities than in gas facilities, so water tunnels have an advantage over wind tunnels. This also results in low Reynolds number, which is convenient for the currently fashionable application to Micro Air Vehicles, and is no particular handicap when studying massive separation from fairly sharp surfaces, where the problem is expected to be largely independent of Reynolds number.

Fundamental questions include the role of vortex formation time ${ }^{1}$ and scaling of aerodynamic force coefficients with motion rate. "Formation time" is the intriguing supposition that a vortex reaches a peak circulation on the basis of energy considerations or kinematic considerations, beyond which the vortex is no longer stable with respect to the solid wall/edge from which its feeding sheet emanates. Maximum circulation is reached, and pinch-off occurs. For aerodynamic applications, one often presumes that the stability of leading edge vortices is important for lift production, and specifically that vortex saturation and pinch-off correlates with peak lift. In the

\footnotetext{
${ }^{1}$ Post-Doctoral Scholar, Air Vehicles Directorate

${ }^{2}$ Senior Aerospace Engineer, Air Vehicles Directorate, Michael.O1@wpafb.af.mil

${ }^{3}$ Associate Professor, Department of Aerospace Engineering.
} 
following, we suggest that this is not necessarily true for very high rates of motion. But this does not mean that a clever definition of the kinematics inviting a universalizing vortex growth/saturation criterion is useless for aerodynamicists. Quite the contrary, such a criterion would allow one to posit the limits of attached-flow and the extent to which management of separated flow, rather than efforts to keep the flow broadly attached, is the preferred recipe for attaining high lift. Even more useful, perhaps, are efforts at scaling lift and drag with respect to motion observables, such as angle of attack and its various derivatives. The ideal is reduction of motion amplitude, rate, acceleration and so forth, towards a simple predictive scaling for lift and drag. For example, Graham and Strickland proposed a phenomenological scaling of lift coefficient by reduced frequency for a 2D airfoil in linear pitch, in an experiment in water ${ }^{2}$. We revisit this model here, for 2D and 3D plates.

Recent work on "perching" motions of 2D flat plates ${ }^{3}$ considered the influence of streamwise deceleration on lift and drag coefficient history, since a perching aircraft intends to land, and the dynamic pressure should go to zero upon maneuver completion. For 2D plates, one finds that the constant-free-stream and streamwise-decelerating cases are not markedly different in lift or drag history, at least until the angle of attack where the constant-free-stream coefficient peak values are achieved. This suggests that while careful study of perching maneuvers does require representation of free-stream deceleration, especially for calculations of stability derivatives and other matters of flight control in the deep-stall regime, the process of vortex formation and shedding can be adequately represented even with a constant free-stream. We reassess this rationale in extending our work on 2D pitching flat plates ${ }^{3}$ and low aspect ratio plates at moderate reduced frequency ${ }^{4}$, to cases of large amplitude and quite high frequency, pitching from zero incidence to $90^{\circ}$.

A second set of pitching cases is based on a set promulgated by the AIAA Fluid Dynamics Technical Committee (FDTC) Low Reynolds Number Aerodynamics Discussion Group (LRADG). It is a smoothed linear ramp beginning at $0^{\circ}$, reaching $45^{\circ}$, holding briefly, and returning back to $0^{\circ}$, all with a pivot at the plate's leading edge. Whereas the 0-90 pitch ramp is concerned mainly with the dynamic stall process, the pitch ramp-hold-return also aims to study return from stall, and lags associated with flow reattachment and the shedding of vortices at the "corners" of the motion - that is, at the beginning and end of the hold, and at the return to zero incidence. Here we aim to compare the $\mathrm{AR}=2$ rectangular plate with results reported earlier for a $2 \mathrm{D}$ plate. ${ }^{9}$

\section{Experimental Setup}

\section{Facility and Motion Mechanism}

Experiments were conducted in the U.S. Air Force Research Lab, Air Vehicles Directorate Water Tunnel. The free-surface tunnel has $4: 1$ contraction and $46 \mathrm{~cm}$ wide by $61 \mathrm{~cm}$ high test section, free-stream speed range of $3-45 \mathrm{~cm} / \mathrm{s}$, and turbulence intensity of $\sim 0.4 \%$ at $15 \mathrm{~cm} / \mathrm{s}$.

The tunnel is fitted with a three degree of freedom motion rig, consisting of a triplet of $\mathrm{H} 2 \mathrm{~W}$ linear motors, driven by AMC DigiFlex servo-drives controlled by a Galil DMC 4040 4-channel card, with user-selected proportional/integral/derivative (PID) constants for each channel. The model's pitch and plunge are controlled via two motors mounted vertically on a plate above the tunnel test section, shown in the left portion of Figure 1. The model's pitch and plunge are controlled via two motors mounted vertically on a plate above the tunnel test section, shown in the left portion of Figure 1. Each motor actuates a vertical "plunge rod", which connects via a bushing to a coupler piece holding the force balance, and ultimately the test article. The upstream plunge rod is constrained to move purely vertically by a nylon track, whereas the downstream plunge rod is allowed to pivot in the test section vertical plane of symmetry. In the present study, the desired motion of the test article is either a pure pitch about some fixed reference point, or a combination of pitch and a fore-aft (streamwise-direction) motion, sometimes termed "surge". Surge is achieved 
using a larger linear motor mounted horizontally aft of the pitch-plunge carriage, with 48" peak-topeak stroke and nominal speed up to $1 \mathrm{~m} / \mathrm{s}$, turning the water tunnel if desired into a towing tank.

The test article's effective pitch pivot point can be varied by suitable choice of position command history of phase and amplitude of the two vertically-mounted linear motors. Commanded vs. attained displacement histories of the three motors are compared by interrogating the three motors' optical encoder tapes, at 5000 increments $/ \mathrm{mm}$ for the two vertical motors, and 1000 increments $/ \mathrm{mm}$ for the streamwise motor. Peak discrepancy between commanded and attained position are $300 \pm 100$ increments, which converts to worst-case of $0.15^{\circ}$ peak error in incidence angle at the plunge rod coupler piece. However, this does not account for vibration or elastic deflection of the plastic coupler piece between the force balance and the model. For all cases where the pitch pivot point is not coincident with the bushed end of the front plunge rod, there will be a parasitic streamwise displacement of the test article, which is removed by introducing corrections in the surge direction. Thus a simple single-degree of freedom motion, pure-pitch, requires the coordinated action of all three motors, but with the advantage that arbitrary changes in pitch fixedpoint are possible without hardware changes.
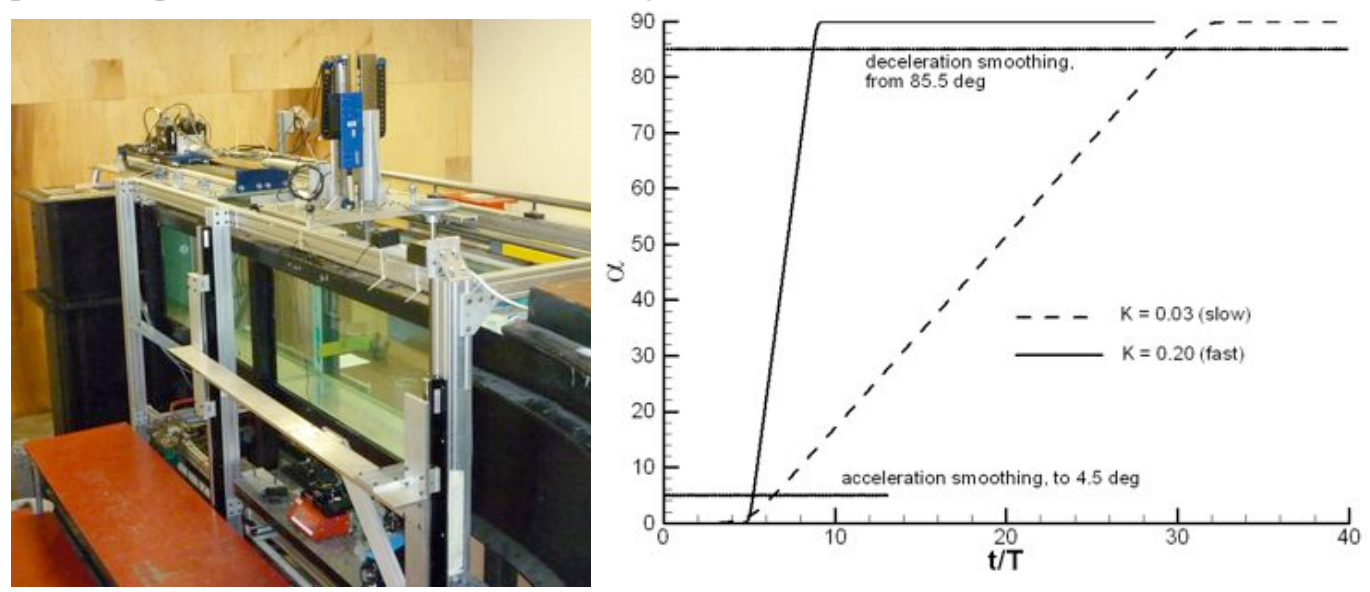

Figure 1. Photograph of water tunnel test section, showing 3-linear-motor motion-rig mounted above test section (left); schematic of pitching motion: angle of attack vs. convective time, for typical "fast" and "slow" pitch rates(right).

\section{Test Article Kinematics and Geometry}

Representative test-article kinematics are given in Figure 1. Pitch motion is nominally constant-rate, or an unaccelerated ramp from $0^{\circ}$ to $90^{\circ}$. But for this to be literally true, pitch acceleration would be a delta function at $\alpha=0^{\circ}$, while pitch deceleration would be a delta function at $\alpha=90^{\circ}$. Instead, smoothing transients are applied. A sine wave of $5 \%$ amplitude $\left(\alpha=4.5^{\circ}\right)$ is tangent to the pitch onset, and an analogous wave tangent to the pitch cessation, is first applied. This smoothes the motion, but not its derivatives. To obtain $\mathrm{C}^{\infty}$ smoothing, a hyperbolic-cosine function suggested by Eldredge et al. ${ }^{5}$, similar in shape to the aforementioned sinusoids, is fitted to the pitch ramp motion. This is shown above and below the dashed lines in Figure 1. Parameter studies on smoothing transients for pitch-ramp motions have been considered before; see for example Koochesfahani and Smiljanovski ${ }^{6}$. Smoothing transients not only make a given nominally constantrate motion realizable in an experiment, but are also important from the viewpoint of noncirculatory or acceleration-dependent aerodynamic forces; this is the critical distinction between the present measurements and the traditional view on the role of startup transients. Noncirculatory forces are due to accelerating the ambient fluid around the moving body, and are not related (at least, not directly) to vorticity production. Noncirculatory forces are zero during the constant-rate portion of the pitching motion, but can be large during the smoothing transients, as they are proportional to the square of the effective motion frequency. In principle, different smoothing transients could be 
applied to the same motion, and different noncirculatory force contributions will result. We also note that since Reynolds number is kept constant $(20,000$ unless otherwise noted) while reducedfrequency of pitch is varied, the physical motion takes more convective times when the reduced frequency is slower. The upper limit for maximum attainable reduced frequency, for a given Reynolds number, is excitation of the model/sting/balance natural frequency, which confounds measurement of noncirculatory aerodynamic forces with oscillations in the apparent aerodynamic force coefficients due to structural vibrations.

Two flat-plate models are considered (Figure 2), both of aspect ratio 2: a rectangular plate and a Zimmerman-planform plate, with ellipses describing the leading edge and the trailing edge, joining along their major-axis on the plate $3 / 4$-chord. Since one would expect that the scaling of leading edge vortices would vary with the local wing chord, vortex evolution along the span should differ between the two plates, for otherwise identical motions. In all cases, the mean aerodynamic chord was used to determine the reduced frequency of pitch, $K=c \dot{\theta} / 2 U_{\infty}$.
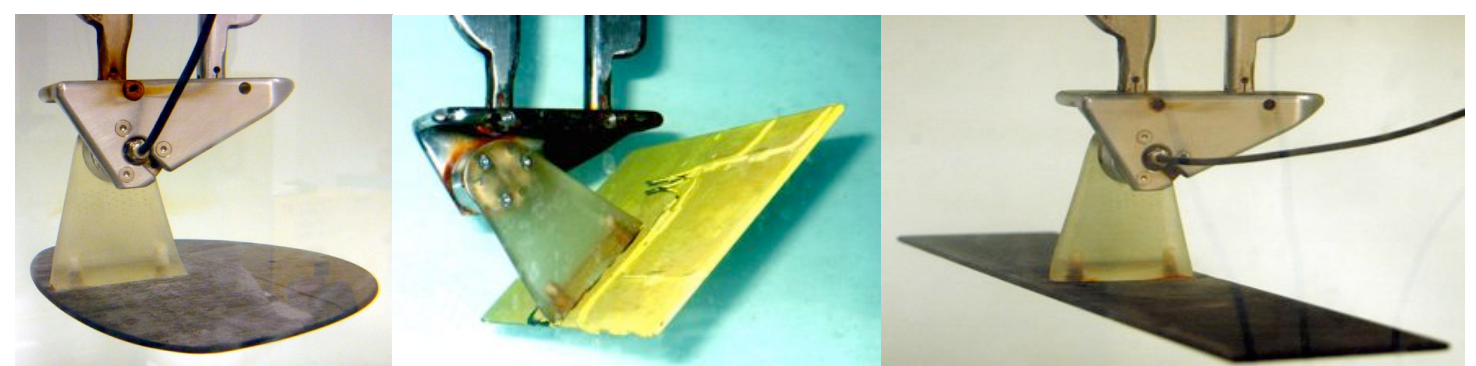

Figure 2. Water tunnel installation of Zimmermann plate (left), rectangular $A R=2$ plate (middle) and earlier case of wall-to-wall plate ${ }^{4}$ (right), at $0^{\circ}, 45^{\circ}$, and $0^{\circ}$ incidence, respectively.

\section{Force Measurement and Flow Visualization}

Dye injection is with a $75 / 25$ (by volume) mixture of blue food coloring and ethanol, to match density with the ambient water. A medical infusion pump introduces dye through stainless steel lines with $0.5 \mathrm{~mm}$ internal diameter, mounted flat against the plate's pressure or suction side. For visualizing leading edge vortices, the dye lines run along the plate leading edge, firing outboard. For visualizing the wake and trailing edge vortices, the dye lines exit in the streamwise direction, terminating just upstream of the trailing edge. And for visualizing tip vortices, dye lines run parallel to the wingtip, firing downstream. Dye is injected at the midspan, 3/4 span, 7/8 span, and near the tip. At low angles of attack, the physical presence of the dye lines disturbs the flow, as wallconformal obstacles. Dye exits at approximately $30 \%$ of the free-stream flow speed. A PCO Dimax digital camera with $24 \mathrm{~mm}$ Nikon lens was used for imaging, operating at 100 frames per second, with illumination by Rosco white LED panels.

Force data are recorded from an ATI Nano-25 IP68 6-component integral loadcell, oriented with its cylindrical axis normal to the pitch-plunge-surge plane. The loadcell is visible in the middle photo in Figure 2. The metric-side of the load cell connects to a plastic vertical strut that bolts to the model. The load cell is offset in the normal direction from the model center of pressure since the forces are much larger in this direction than in axial. This setup is much stiffer than a sting mount with the loadcell mounted downstream of the wing without producing any significant difference in flow interference during dynamic testing ${ }^{4}$. This is the alternative to a more geometrically intrusive mount, where the balance is placed directly at the model center of pressure. A disadvantage of the IP68 waterproofing of the loadcell is that it is sensitive to immersion depth in the cylindrical axis direction. Because this direction is normal to the plane of the motion of symmetrical models, the hydrostatic force will not affect normal force, axial force or pitching moment. Force and motion data are synchronized by polling for the trigger signal every $10 \mathrm{~ms}$ and starting the data recording when initial trigger is detected. 
Load cell strain gage electrical signals are A/D converted in an ATI "NetBox" interface and recorded over Ethernet LAN UDP protocol to a computer using a Java application, and are filtered in three steps. This first is a low-pass filter in the ATI NetBox at $\mathrm{f}=73 \mathrm{~Hz}$, to avoid introducing noise not correlated with motion force data, but without attenuating important fast non-circulatory "load spikes". These are on the order of a fifth as slow as the $-3 \mathrm{~dB}$ (half amplitude attenuation) point of the filter for the fastest pitch ramp corner acceleration. The second step uses a moving-average of 11 points to smooth the data while preserving as much of the non-circulatory load spikes as possible. This smoothing also makes more numerically stable the final step, which is a $4^{\text {th }}$ order Chebychev II low-pass filter with $-20 \mathrm{~dB}$ attenuation of the stopband. The cutoff frequency is five times the motion frequency. It is chosen for maximum passband flatness and high rejection of structural eigenfrequencies, which may be just above the desired force frequency information range. To preclude time-shift of useful data in the passband, the forward-backward filtering technique with the Matlab 'filtfilt' command is used.

\section{Tare Subtraction and Uncertainty Analysis}

Before each run, the load cell is zero-biased at model $\theta=0^{\circ}$, which is adjusted to horizontal with a bubble level. Depending on blockage effects from the sting, zero geometric incidence angle may not correspond to zero lift. A static tare sweep over $0^{\circ}<\theta<90^{\circ}$, is performed with 500 samples of data every $2^{\circ}$. Because the pitch angle is known throughout the motion, and the position error is negligible, the static axial force, normal force and pitching moment due to static model $/ \mathrm{sting} / \mathrm{mount}$ weight can be subtracted from the unsteady force data. Figure 3 offers a convenient shortcut in static tare subtraction, as evidently the axial force, normal force and pitching moment coefficient are each linear with $\sin (\theta), 1-\cos (\theta)$, and $\sin (\theta)$, respectively.

The most pedantic approach to tares is a combination of static and dynamic: the "true" hydrodynamic force equals the dynamic measurement in water, minus the respective static measurement in water, minus the difference between the dynamic measurement in air, and the static measurement in air. So there are two dynamic runs - with the water flowing, and with the test section drained of water; and two static tare sweeps, in water and with the water drained. We consider the relative merits of this approach, vs. the simpler approach of completely ignoring static and dynamic runs with the water drained, for one of the LRADG linear pitch ramp-hold-return maneuvers introduced above.
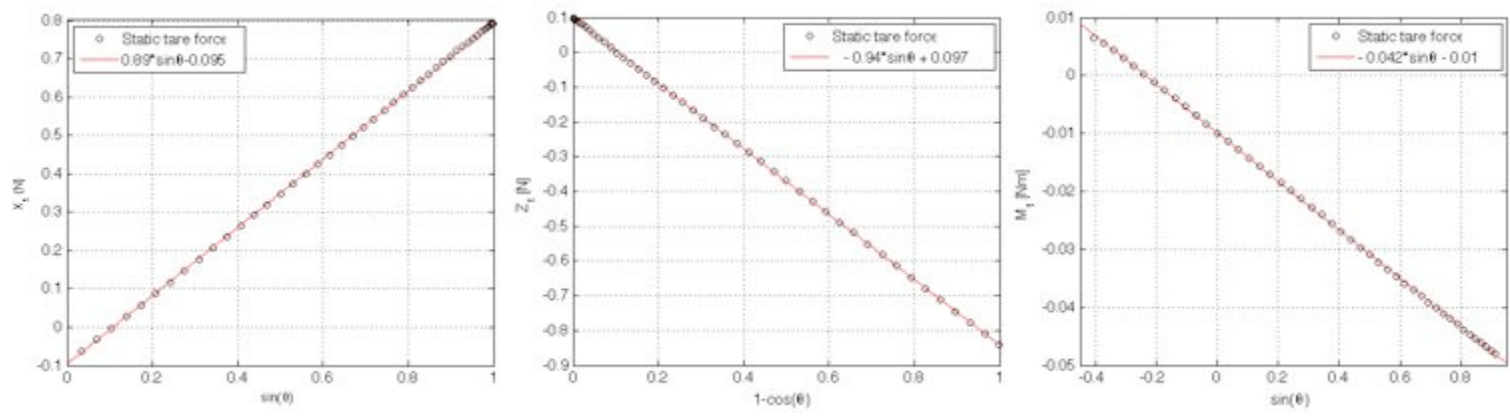

Figure 3. ATI Nano-25 load cell post-processing: Linear fit to tare axial- (left), normal (middle) force and pitching moment (right)

Figure 4 compares lift and drag data amongst four ingredients: dynamic data in water (that is, the $0^{\circ}-45^{\circ}-0^{\circ}$ pitch maneuver), dynamic data in air (same maneuver with the water tunnel test section drained), and the two static sweeps, in water and air. While none of the three tares are with water flowing, all are reduced to "aerodynamic coefficients" by assuming the same dynamic pressure as in the dynamic run in water. This allows for comparison of relative amplitudes using 
consistent units. Static and dynamic data in air are seen to be very close, for both lift and drag. This suggests that acceleration effects for the model itself are benign. The "lift" dynamic tare in air has slight bumps at motion initiation and cessation; these are the plate rigid-body acceleration loads, and are seen to be small in relative terms. For lift, the dynamic run in water has much larger amplitude than the static tare in water, and is larger than the runs in air. But for drag, the dynamic run in water is of somewhat smaller than the static tare in water, and both are smaller than the runs in air.

The models are made of carbon fiber, and therefore buoyancy effects are significant. That, is the static are magnitude in air will be much larger than in water. The dynamic-static tare subtraction in air is the subtraction of two large but quite comparable numbers. For this reason, using static and dynamic tares in air is as likely to introduce additional error, as to remove it. In the following, we ignore the four-way tare procedure entirely, and simply subtract the static tare in water from the dynamic run in water.
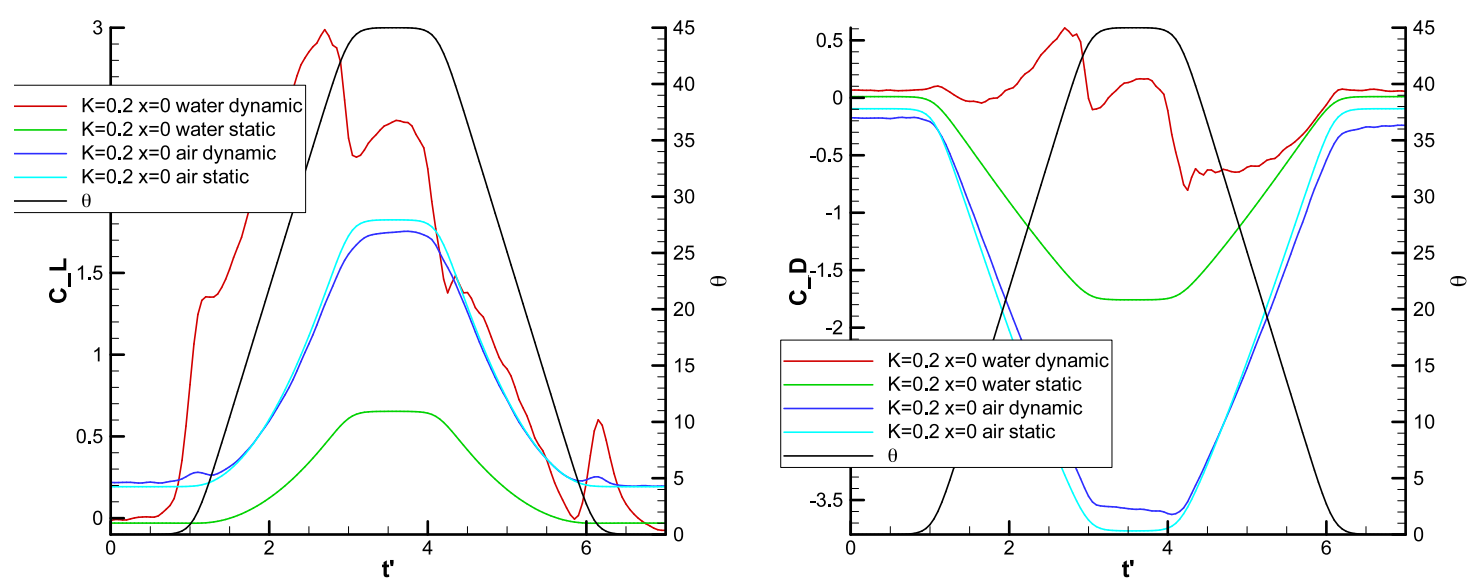

Figure 4. Example of static and dynamic tare procedure, lift (left) and drag (right), for FDTC LRADG 0-45-0 pitch ramp-hold-return motion.

\section{Results}

We first present sequences of flow visualization snapshots for LEV, TEV and tip-vortex evolution for the rectangular and Zimmermann plates, for high-frequency $0^{\circ}-90^{\circ}$ smoothed linear pitch ramps. Next, we survey lift and drag coefficient history for $0^{\circ}-90^{\circ}$ pitch ramps, in two conditions: in the usual case with constant free-stream, and with decelerating free-stream or "surge". Then we examine pitch ramp-hold-return problems continuing the AIAA Fluid Dynamics Technical Committee's "Low Reynolds Number Aerodynamics Discussion Group's" canonical cases, for the $\mathrm{AR}=2$ rectangular plate. Finally, we present an attempt at scaling lift coefficient following Graham and Strickland ${ }^{2}$, in the hope of elucidating explicit relationship of lift to pitch frequency.

\section{Flow Visualization}

Flow visualization results for $\mathrm{K}=0.50$ are given for the rectangular plate in Figure $5 . \mathrm{K}=$ 1.0 cases are given in Figure 6 and Figure 7 for the rectangular plate and Zimmerman plate, respectively. Figure 6 compares pitch pivot points of $\mathrm{x} / \mathrm{c}=0$ (leading edge), $\mathrm{x} / \mathrm{c}=0.5$ (midchord) and $\mathrm{x} / \mathrm{c}=1.0$ (trailing edge), while Figure 5 and Figure 7 are limited to $\mathrm{x} / \mathrm{c}=0$ and 1.0. Side views, showing LEV and TEV sectional cuts, are compared with planform views, which show spanwise development of the LEV, TEV and tip vortex. This approach follows that of Yilmaz et al ${ }^{7}$., where spanwise and chordwise development of LEVs was compared for an $\mathrm{AR}=2$ plate in a pitch ramphold-return motion. 
In comparing with Granlund et al. ${ }^{3}$, which discuss results for identical motions but for a 2D plate, the LEV for all $\mathrm{AR}=2$ cases is seen to be less coherent, in the sense of greater dye diffusion and less dye entrainment into a definitive vortex core. In comparing LE, midchord and TE pivot points, one finds that LEV development is delayed when the pivot point is taken to be further aft. Indeed, for the larger pitch rates and the TE pivot point, an LEV first forms on the plate pressure (receding) side, before switching to the suction (advancing) side. Spanwise flow from inboard towards the centerplane, as identified in Yilmaz et al. ${ }^{7}$, is strongly present for the rectangular plate, and is apparent in the LEV as soon as it forms. For the Zimmerman plate in Figure 7, this spanwise flow is much weaker than for the rectangular plate, possibly because for the former the spanwise variation of chord results it its own spanwise pressure gradient effect. Dye does not venture far spanwise from its ejection point until well into the LEV's development.

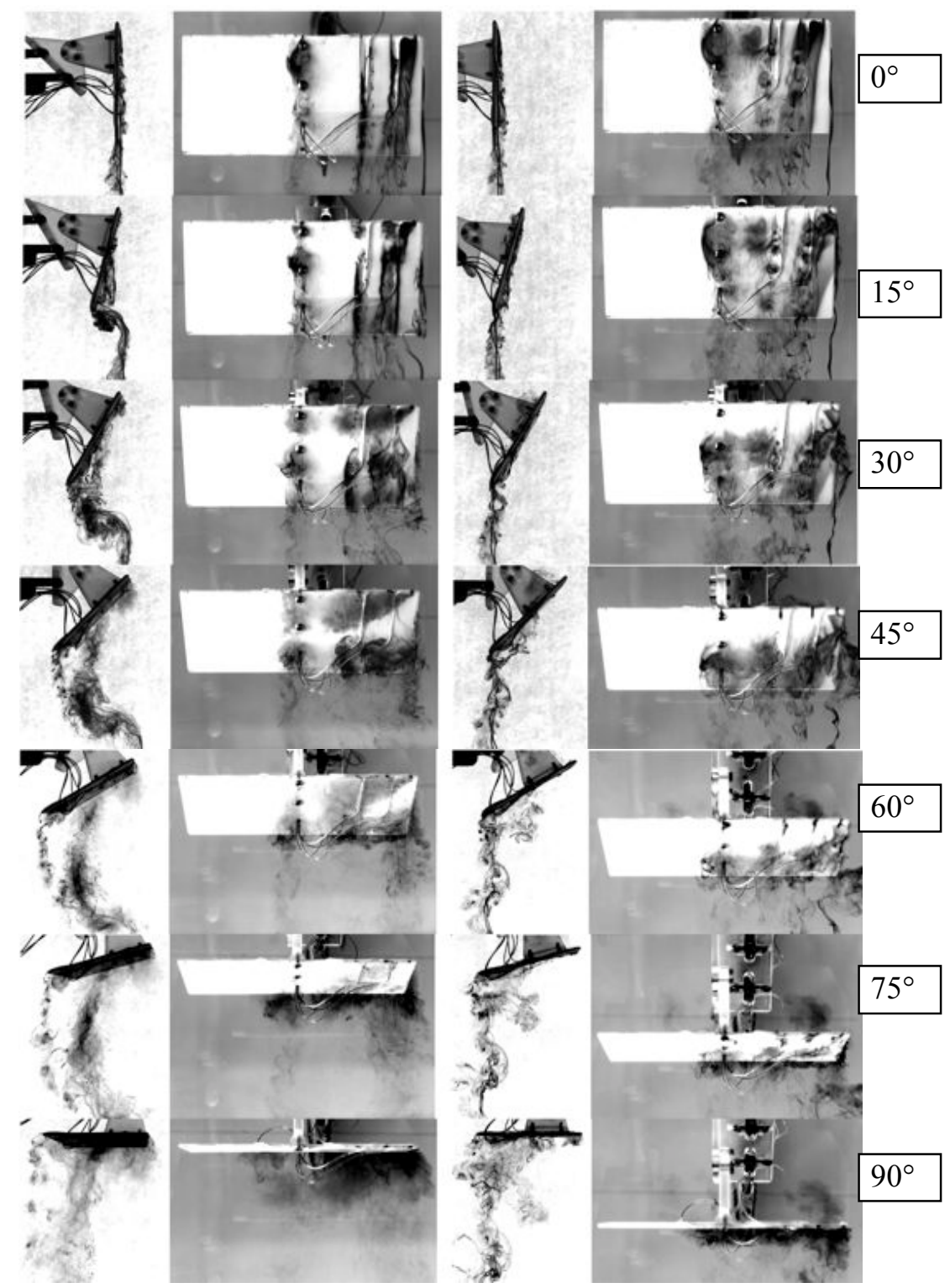

Figure 5. Side view and planform view of rectangular $A R=2$ plate pitching at $K=0.50$, with pivot at leading edge (left) and trailing edge (right). Snapshots at incidence angles of $0^{\circ}, 1^{\circ}, 30^{\circ}, 4^{\circ}, 6^{\circ}, 7^{\circ}$ and $90^{\circ}$. 

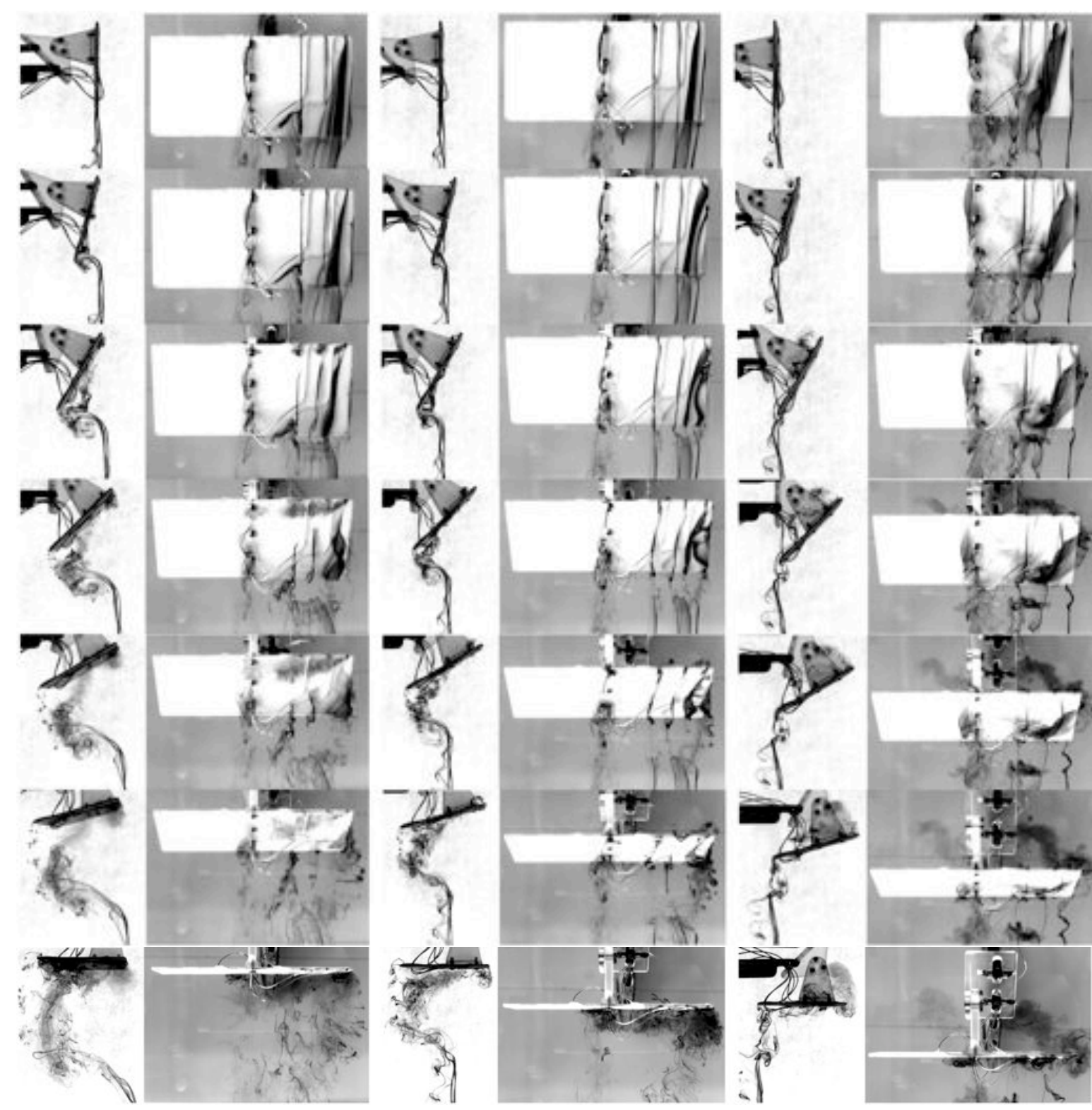

Figure 6. Side view and planform view of rectangular $A R=2$ plate pitching at $K=1.0$, with pivot at leading edge (left), midchord (middle) and trailing edge (right). Snapshots at incidence angles of $0^{\circ}, 15^{\circ}$, $30^{\circ}, 45^{\circ}, 60^{\circ}, 75^{\circ}$ and $90^{\circ}$.

The tip vortex is seen to deflect with increasing angle of attack at a slower rate than LEV evolution, in the sense that tip vortex trajectory remains parallel relative to the wing chord-plane to angles of attack by which the LEV has already formed, and indeed detached. This suggests, at least notionally, that tip vortex formation-time, in dimensional terms, is longer than LEV formation time. Since a tip vortex is a necessary and sufficient condition for nonzero bound vorticity, the tip vortex's hypothesized slow evolution corroborates the assertion that bound vorticity remains significant to high angles of attack, provided that reduced frequency of pitch is high enough. The further implication is that the leading edge vortex, or more properly the vortex system of which the LEV is a part, is a scheme of retaining significant bound circulation in an otherwise massively separated flow.

Increasing the reduced frequency is seen to tighten the LEV and to delay its formation to higher plate incidence angles, such that in the case of aft-most pivot, the LEV has not reached its maximal extent until after the motion is completed. As will be seen in the next section, peak 
circulatory lift is achieved sometime around the midpoint of the pitching motion, and never towards the conclusion of the motion, regardless of the pitch rate or pivot point location. Therefore in cases of sufficiently high reduced frequency - such as $\mathrm{K}=1.0$ and above - the LEV can not be the main direct contributor to high lift.

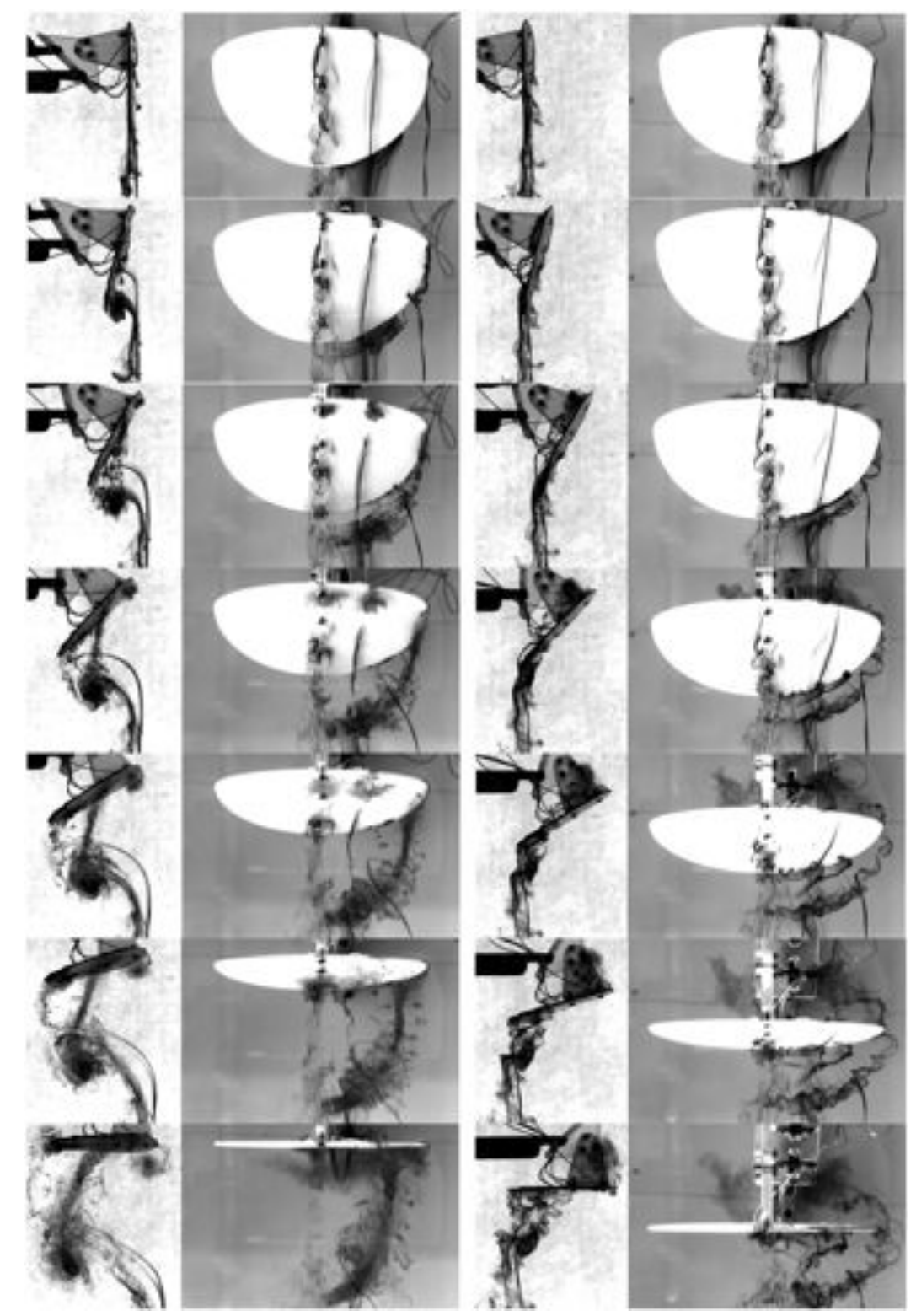

Figure 7. Side- and planform view of Zimmerman $A R=2$ plate pitching at $K=1.0$, with pivot at leading edge (left) and trailing edge (right). Snapshots at incidence angles of $0^{\circ}, 15^{\circ}, 30^{\circ}, 45^{\circ}, 60^{\circ}, 7^{\circ}$ and $90^{\circ}$.

Besides differences in the LEV spanwise development between the Zimmerman plate and rectangular plate, the interaction between tip vortex and TEV are also quite different. For the rectangular plate, the tip vortex remains more or less aligned with the free stream in the planform view, and is distinct from the trailing edge vortex. For the Zimmerman plate, the tip vortex wraps around the trailing edge and merges with the trailing edge vortex, evidently enhancing the latter's stability. As will be seen next, differences in the flowfields between the two wings are much larger than differences in the measured lift and drag coefficients. This suggests, again, that bound vorticity, and not the induction of off-body vortical structures, is the dominant source of circulatory forces. 


\section{Lift and Drag Measurements for $0^{\circ}-90^{\circ}$ Pitch Ramps}

For low reduced frequency, one expects benign departures in lift and drag history from the static case; that is, the response should be quasi-steady. As pitch rate increases, "dynamic stall" manifests itself as a continuation of the steady lift curve to angles of attack progressively higher than that of static stall, and the stall nose-over is smoothed out. The actual stall angle corresponds closely with the angle of attack range where the LEV achieves maximal circulation and begins its pinch-off and shedding process. Granlund et al. ${ }^{4}$ showed this to be the case for $\mathrm{K}<0.3$. For even higher reduced frequencies, correlation between peak lift and LEV saturation is less obvious, and noncirculatory effects, which begin to manifest themselves at $\mathrm{K} \sim 0.1$, can become very large. We explore these themes below.

\section{2.a. Reduced-Frequency Parameter Studies}

Here our purpose is to extend the results of Granlund et al. ${ }^{4}$ to $\mathrm{K}=1$. Lift and drag results for $\mathrm{K}=0.1$ through $\mathrm{K}=1.0$ for the $\mathrm{AR}=2$ rectangular plate are compared in Figure 8, while the rectangular and Zimmerman plate are compared in Figure 9, all for a pivot point of $\mathrm{x} / \mathrm{c}=0.25$ (quarter-chord).

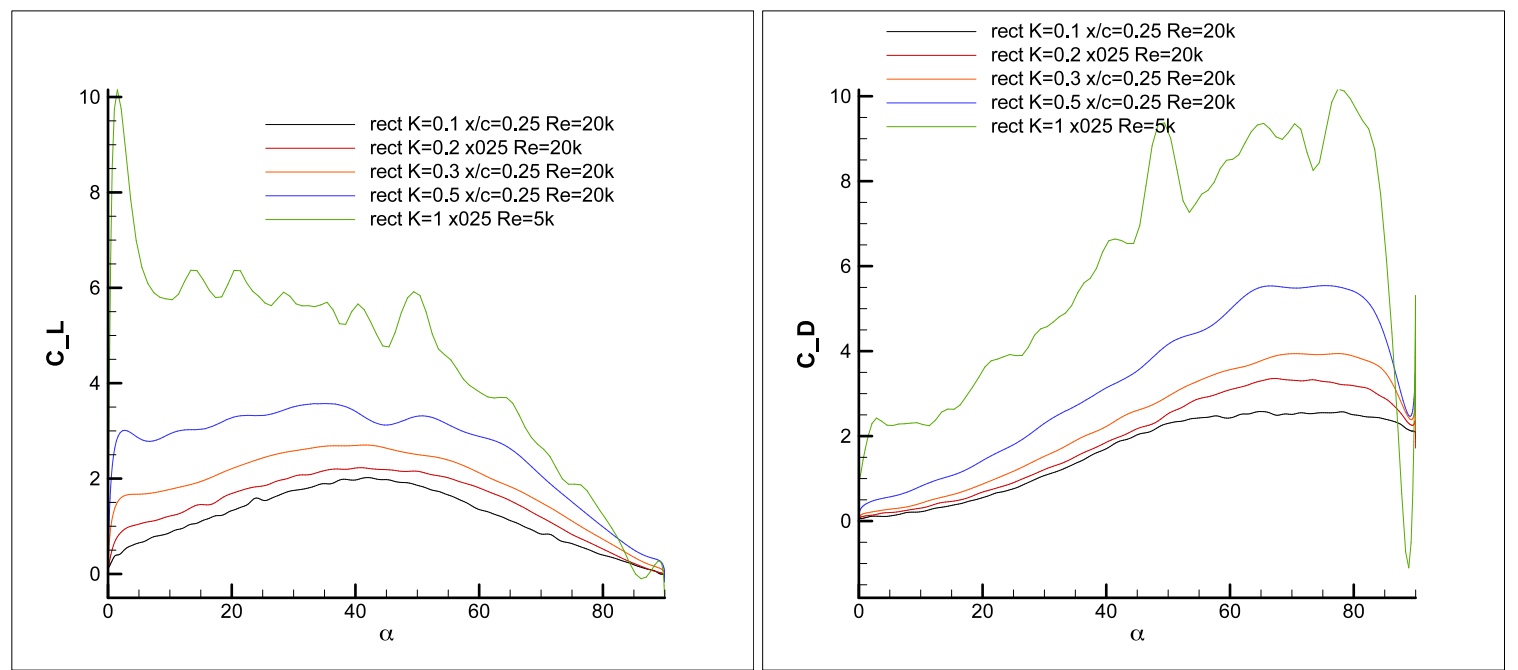

Figure 8. Lift (left) and drag (right), $K=0.1$ through 1.0, $A R=2$ rectangular plate, pivot about $x / c=0.25$. $K=1.0$ data are at $\operatorname{Re}=5000 ;$ all others are at 20,000 .

Both lift and drag evince monotonic increase with increasing reduced frequency. The $\mathrm{K}=$ 1.0 curves are notable for the large noncirculatory load spikes: a strong positive spike in lift just above zero incidence, and a similarly strong negative spike in drag at 90 degrees incidence. Both occur strictly during the smoothing transients, where angular acceleration is nonzero. Similar, but smaller, noncirculatory spikes are seen for $\mathrm{K}=0.5$, but for smaller frequencies these spikes become difficult to distinguish, possibly because they have force histories appear to have a proportionality with $\mathrm{K}$; this is explored in Section 4 below, where we discuss scaling relations. One fairly clear departure from strict proportionality of lift with reduced frequency is the apparent decrease in lift slope beyond the initial spike.

The Zimmerman plate is seen to have similar force histories to those of the rectangular plate, with generally lower lift and drag in all cases. This is perhaps surprising, in light of the differences in flow visualization between the two. 


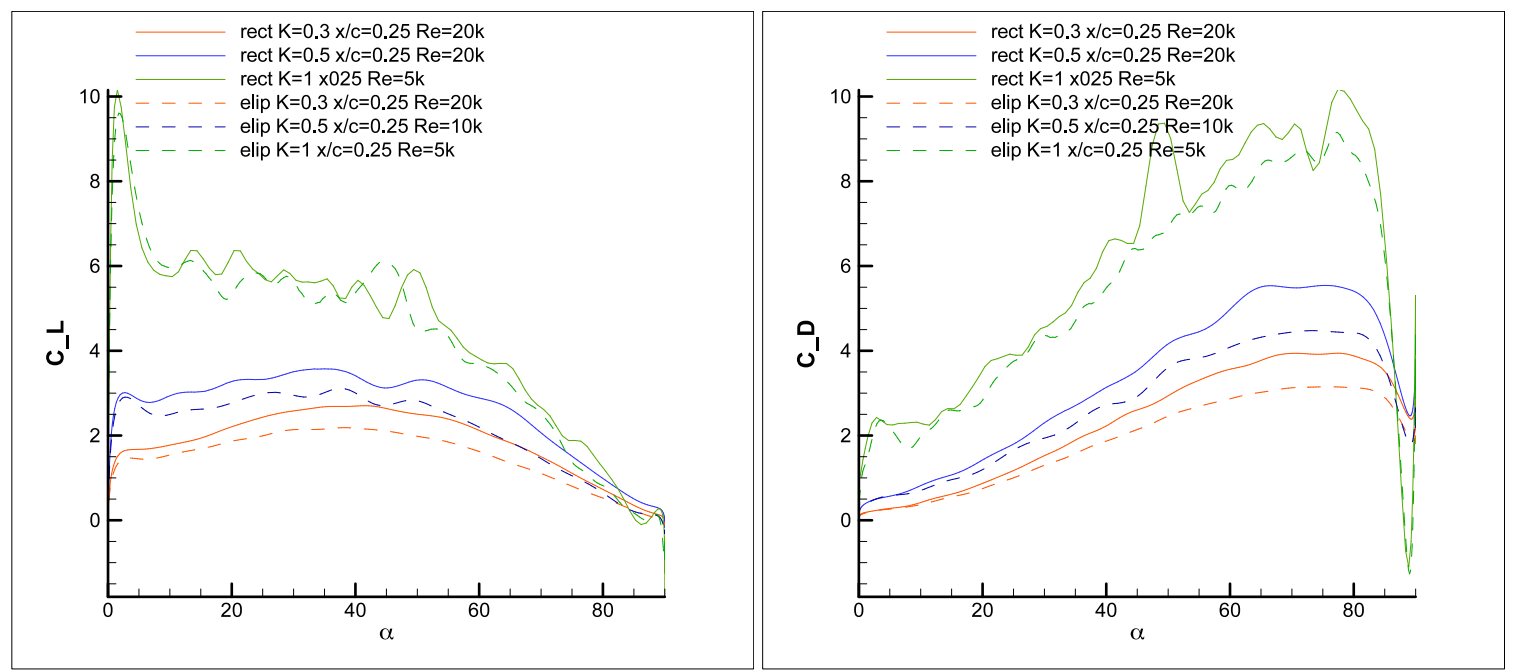

Figure 9. Comparison of $A R=2$ rectangular and $Z$ immerman plates for $K=0.3,0.5$ and 1.0, pivoting about $\mathrm{x} / \mathrm{c}=0.25$; lift (left) and drag (right).

The angle of attack corresponding to the peak lift coefficient appears to become smaller with increasing reduced frequency. This is the opposite of the trend observed by Granlund et al. ${ }^{3}$ for $2 \mathrm{D}$ plates for $\mathrm{K}+0.2$ and below. Drag, on the other hand, peaks at $\sim 75^{\circ}$ for all cases.

\section{2.b. Effects of Pivot Point Location}
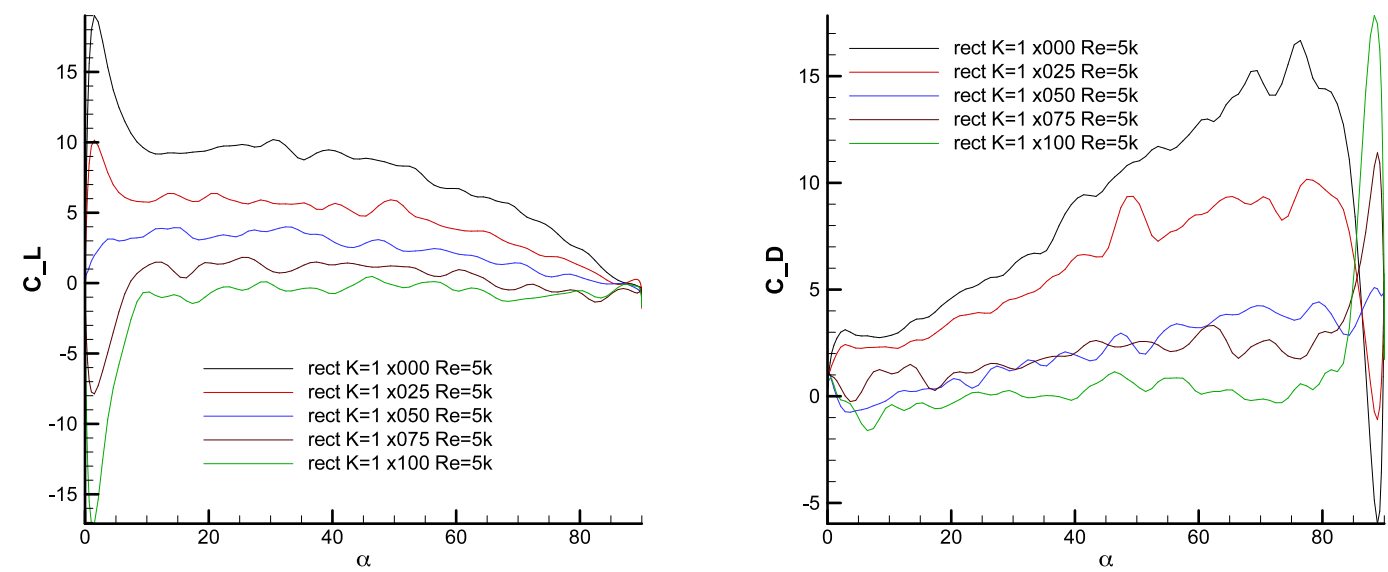

Figure 10. Effect of pitch pivot point $(x / c=0,0.25,0.50,0.75$, and 1.0$), A R=2$ rectangular $p l a t e, K=1.0$.

Dye injection results showed a marked dependency of flowfield evolution on the pitch pivot point location. LEV development on the plate's suction side is lagged as the pivot point is taken to be further aft, and the immediate question is whether this lag is observable in the lift and drag history. Figure 10 shows pivot point effects for $\mathrm{K}=1$ for the $\mathrm{AR}=2$ rectangular plate. The main effect of different pivot point is not a lag in aerodynamic response, but a huge difference in the sign and direction of the transients during the smoothed portion of the linear pitch ramp (starting and stopping transients). For $\mathrm{x} / \mathrm{c}=0$ pivot, the initial lift spike is $\mathrm{C}_{\mathrm{L}} \sim 18$, while for $\mathrm{x} / \mathrm{c}=1.0, \mathrm{C}_{\mathrm{L}} \sim-18$. As theory predicts, for $\mathrm{x} / \mathrm{c}=0.5$, the initial spike is absent. These are all strictly noncirculatory effects, as this is where acceleration occurs in the smoothing transient between rest at the linear pitch ramp. During the unaccelerated portion of the pitch ramp, the different lift curves have an almost 
constant offset between them, with a small slope change that drives all curves ultimately to zero lift at 90 degrees incidence. In drag, this slope difference is much more pronounced, culminating in the noncirculatory spike towards 90 degrees incidence and eventually relaxation to a steady-state drag coefficient near 1.5 .

\section{2.c. Effects of "Surge"}

Granlund et al. ${ }^{3}$ compared 2D flat plate results for a constant-free-stream linear pitch ramp, with that of constant streamwise deceleration, or so-called "surge". Motion with surge attempts to mimic, or at least to idealize, the scenario relevant to a "perching" maneuver", where the aircraft decelerates in approaching a landing, as well as undergoes pitch-up. One asks how the resulting increase in reduced-frequency, as the relative free-stream speed is reduced, affects the history of lift and drag coefficients with respect to angle of attack. For a 2D flat plate, Granlund et al. ${ }^{3}$ found minimal effect of surge until well into deep stall, say 50 degrees angle of attack. The lift and drag coefficients were obtained from instantaneous normalization, where one takes the instantaneous dynamic pressure from the plate-to-free-stream relative speed as a function of time, to form the aerodynamic force coefficients. As the pitch maneuver progresses, the resulting dynamic pressure declines to zero as the incidence angle approaches 90 degrees, whereupon the meaning of the aerodynamic coefficients becomes moot. First, at very small dynamic pressure such normalization is essentially a division by zero. Second, the force balance signal to noise ratio becomes unacceptably small and therefore the data become suspect. Nevertheless, the attraction of this approach is that the with-surge and no-surge pitch at the same nominal reduced frequency can be compared directly. Presently we follow the same approach for the $\mathrm{AR}=2$ wings.
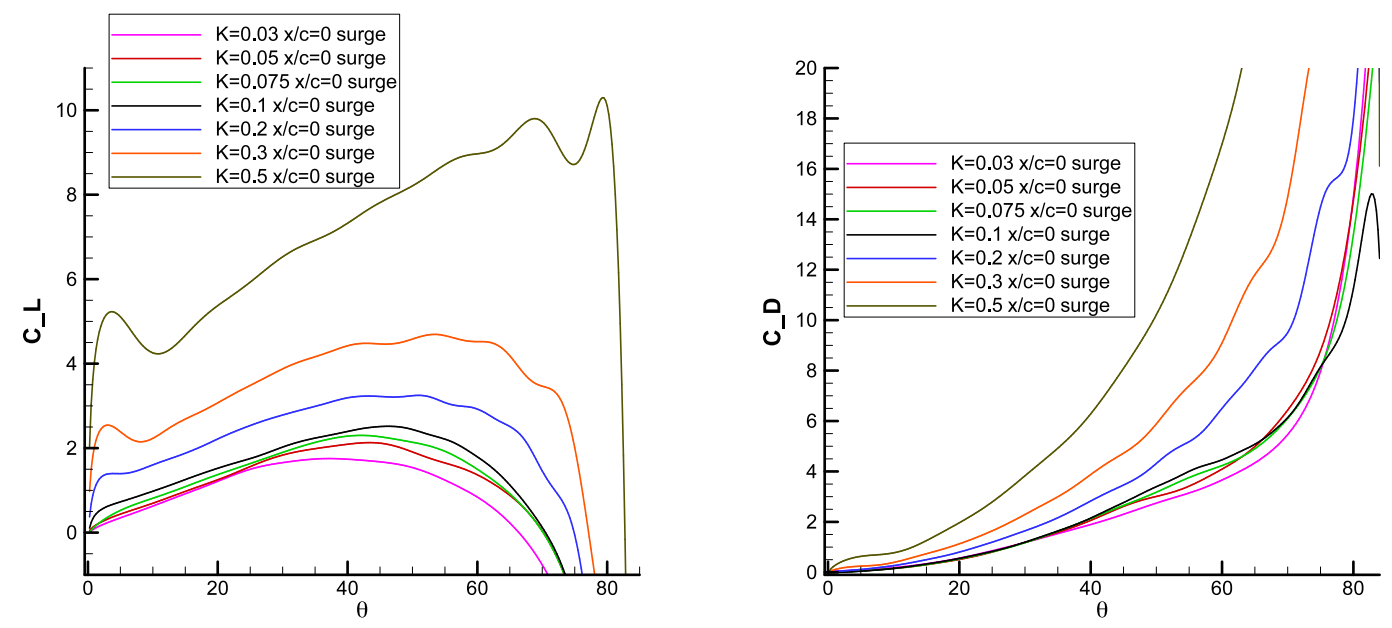

Figure 11. Survey of reduced frequency with "surge", $A R=2$ rectangular plate, pivot point $x / c=0$.

Figure 11 shows lift and drag results for a range of reduced frequencies for the $A R=2$ rectangular plate pitching about $\mathrm{x} / \mathrm{c}=0$, while Figure 12 compares the respective with-surge and without-surge cases for pivot about $\mathrm{x} / \mathrm{c}=0.25$. The leading edge pivot point is perhaps the most interesting, as this produces the highest lift. As compared with the 2D plate, the surge vs. no-surge difference is somewhat larger for the $\mathrm{AR}=2$ plate, but even so, the solid and dashed curves in Figure 12 are much alike, with differences progressively larger for larger reduced frequency. With surge, forces are higher than without, because the effective reduced frequency increases. The noncirculatory lift spike at motion onset is essentially identical in all cases, as is to be expected, since the with-surge and no-surge cases are at the same free-stream speed at pitch initiation. The low angle of attack agreement between surge and non-surge cases can also be viewed as a secondary check on data repeatability. And as with the $2 \mathrm{D}$ case, force coefficients become unstable (drag 
actually becomes arbitrarily large in magnitude and negative) for large incidence angles. This is partially because of the mechanical realization of the experiment in the water tunnel. With-surge cases are run starting the model far downstream in the test section, by rapidly accelerating the horizontal linear motor to oppose the test section flow speed, such that the difference between the two speeds gives the nominal desired Reynolds number for the "cruise" portion before the maneuver. The velocity is then held constant for ten convective lengths to remove startup transients. For the actual pitching motion, the rig decelerates in going upstream while the pitch angle increases, until the rig stops near the upstream end of the test section where the net velocity is now reduced to half of the initial, and the pitch angle is $45^{\circ}$. The rig continues its deceleration and begins to move back downstream until reaching a pitch angle of $90^{\circ}$ where its streamwise speed matches the tunnel flow speed, for zero net free-stream. The net zero velocity is held constant for the wall clock time it would take to cover five convective lengths at initial conditions. Any slight mismatch in speeds will produce a fictitious dynamic pressure where none is intended. Additional uncertainty, resulting in spurious aerodynamic force coefficients late in the maneuver, is from departures from nominal freestream flow speed far downstream in the test section, vs. further upstream. Nevertheless, the high angle of attack behavior of the surging $A R=2$ plate is much the same as that of the $2 \mathrm{D}$ plate ${ }^{3}$, reaffirming the relative unimportance of realizing a free-stream deceleration in addition to the pitchramp, in modeling a perching maneuver.
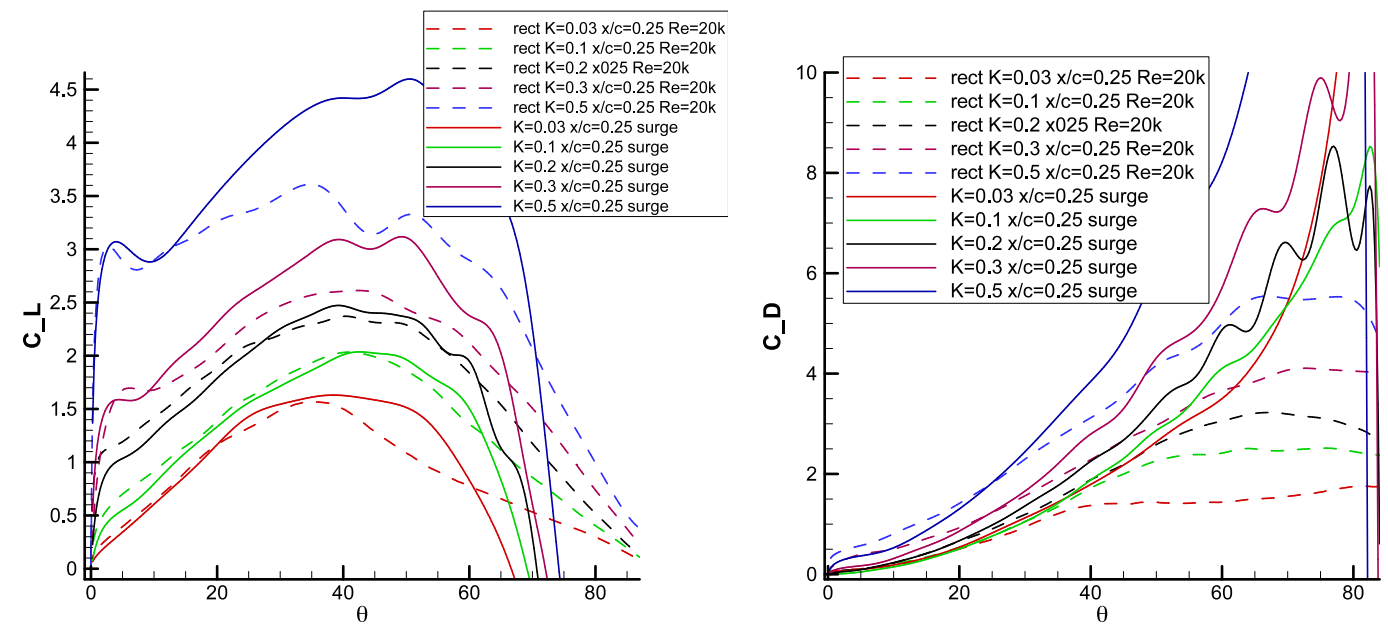

Figure 12. Comparison of constant relative free-stream (solid lines) and decelerating relative free stream ("surge", dashed lines), $\mathrm{AR}=2$ rectangular plate, pivot point $\mathrm{x} / \mathrm{c}=\mathbf{0 . 2 5}$.

\section{2.d. The Case of No Free Stream}

The limit of infinite reduced frequency can intuitively be realized by turning off the water tunnel completely. Now the physical rate of motion determines a kind of Reynolds number, and the ratio of hydrodynamic forces to components of the model's weight. With no flow, one expects shed vortices to not convect downstream with a baseline speed (that is, the free-stream speed), and instead to only move under self-induction of the vortex system. Therefore, nonzero lift should persist for more convective times than for the case of free-stream. 

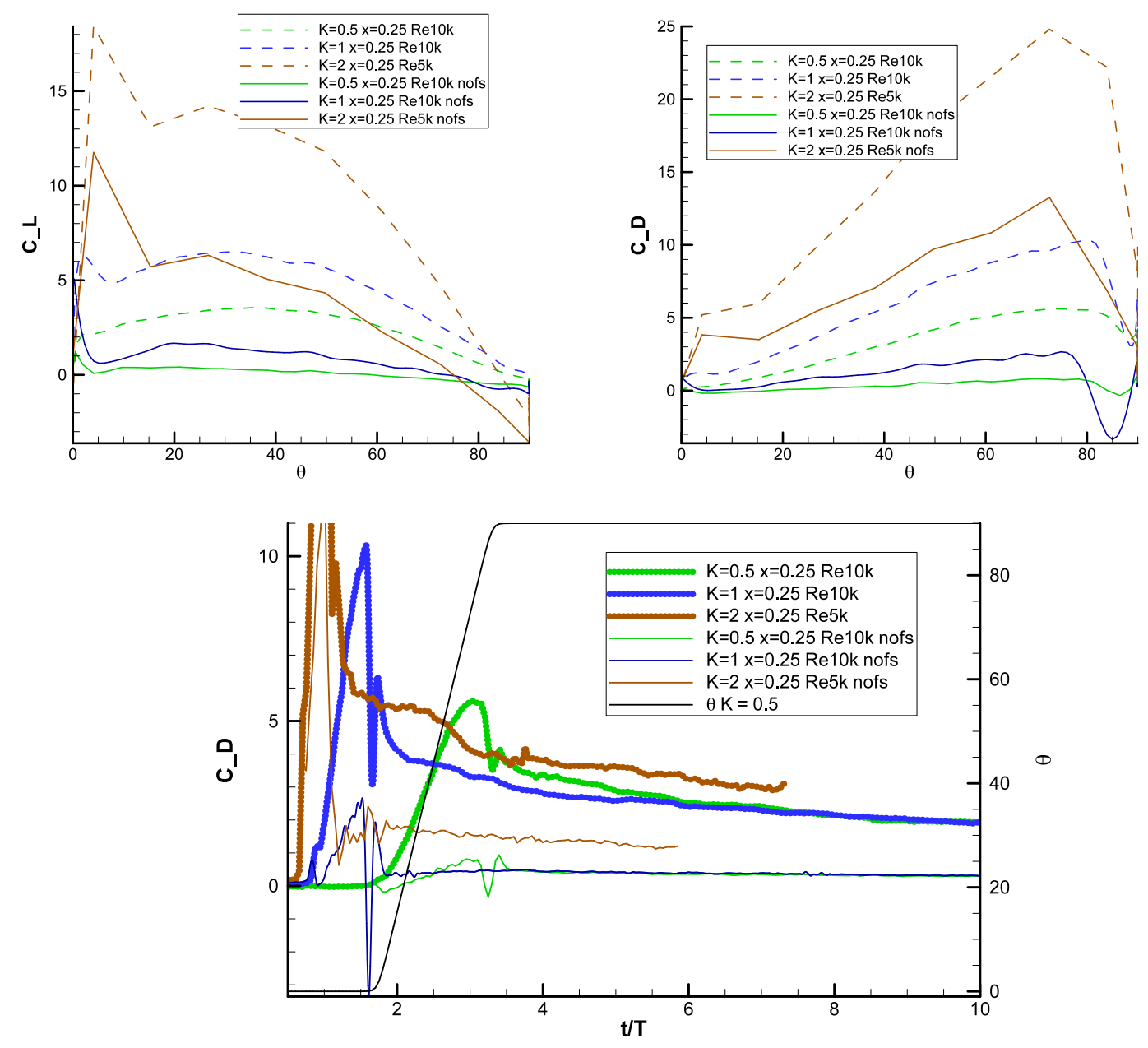

Figure 13. Lift (left) and drag (right) for $A R=2$ rectangular plate, with flow (dashed lines and without flow (solid lines). Lift is plotted vs. angle of attack, while drag is plotted vs. convective time, together with angle of attack history for the $K=0.1$ case.

Figure 13 compares with-flow cases of $\mathrm{K}=0.5,1.0$ and 2.0, with the respective no-flow cases, where the latter are the same motion as with-flow, but with the water tunnel turned off. Even at this high reduced frequency, the difference between the two families of cases is striking. For $\mathrm{K}=$ 2 , the low-incidence noncirculatory spikes begin to become comparable, but the circulatory lift in the no-flow case is much lower than in the case with flow. Evidently, the dynamic pressure of the flowing water is essential for producing large aerodynamic force coefficients.

The drag coefficient in Figure 13 is plotted both against angle of attack and convective time, with the motion history for $\mathrm{K}=0.5$ also plotted for reference. The operative questions in plotting drag against convective time are, first, how long does it take to relax towards the drag value expected for a flat plate statically mounted at 90-degrees incidence to the flow; and second, how many convective times are required before the eventual onset of periodicity in drag, due to Karman shedding. Broadly, one finds that the higher the reduced frequency, the longer the relaxation in drag. In no case was a drag oscillation indicative of Karman shedding achieved, even by $t / T=10$. And in no case was there sufficient time for relaxation to the static drag value of approximately $C_{D} \sim 1.5$.

A similar comparison of with-flow and no-flow cases is shown in Figure 14, this time for the $2 \mathrm{D}$ plate. The $2 \mathrm{D}$ plate was chosen instead of the $\mathrm{AR}=2$ plate because it has more coherent and 
more obvious LEV and TEV structure. At $K=1$, the noncirculatory spikes in lift at low angle of attack are again similar for the with-flow and no-flow cases. But as with the $A R=2$ plate, the circulatory contributions are far apart. We conclude that a frequency much higher than $\mathrm{K}=1$ would be necessary to render small the aerodynamic response to free-stream dynamic pressure relative to the effect of plate rotation itself.
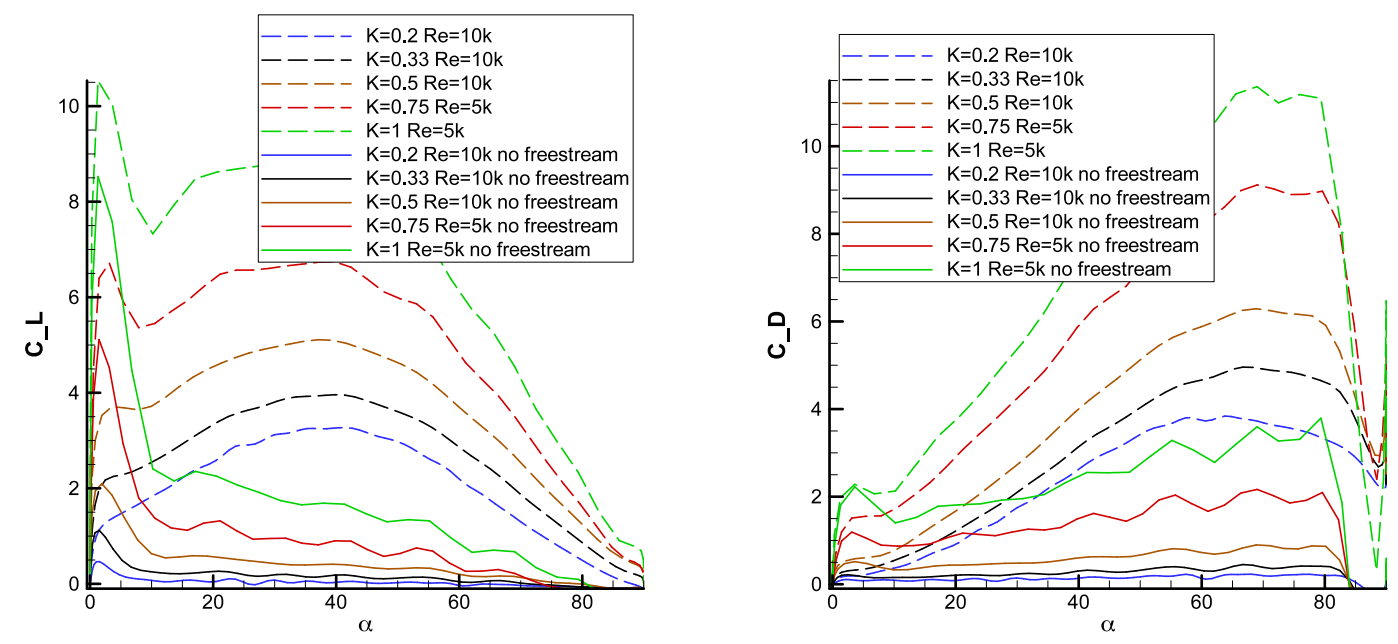

Figure 14. Lift (left) and drag (right), 2D plate, with and without flow, for reduced frequency from 0.2 through 1.0; Reynolds number as marked. Pitch pivot point is $x / c=0.25$.

\section{Lift and Drag Measurements for the Canonical Pitch Ramp-Hold-Return Problem}

The AIAA Fluid Dynamics Technical Committee (FDTC) Low Reynolds Number Aerodynamics Discussion Group (LRADG) has been pursuing "canonical problems" of a flat-plate pitch-ramp-hold maneuver, akin to the linear pitch ramp studied above, but with the added complication of time spent by the plate at its maximal incidence, and a return to zero incidence. The hold-time at maximum incidence is such that the "trapezoid" of the ramp-up, hold- and ramp-down has the upstroke and downstroke tangent to a sinusoidal motion of the same amplitude and reduced frequency. Thus far, only the $2 \mathrm{D}$ case was considered, in a motion of $0^{\circ}-45^{\circ}-0^{\circ}$. The main case featured $\mathrm{K}=0.2$, from an interest in moderate-frequency effects. Pivot is about the leading edge, as this has been shown to produce maximal lift and drag. To this we add a "slow" case, $\mathrm{K}=0.03$, to have more relevance to perching maneuvers, and to be more amenable to testing in wind tunnels and not just in water tunnels. The current experimental force measurements for the $\mathrm{AR}=2$ rectangular plate are therefore: $\mathrm{K}=0.2$ and $0^{\circ}-45^{\circ}-0^{\circ}$ (Figure 15), $\mathrm{K}=0.2$ and $0^{\circ}-90^{\circ}-0^{\circ}$ (Figure 16), $\mathrm{K}=0.03$ and $0^{\circ}-45^{\circ}-0^{\circ}$ (Figure 17), and $\mathrm{K}=0.03$ and $0^{\circ}-90^{\circ}-0^{\circ}$ (Figure 18), all using the kinematics and smoothing-functions reported in $\mathrm{Ol}$ et al. ${ }^{9}$ and with the initial ramp corner at $\mathrm{t} / \mathrm{T}=1$. 

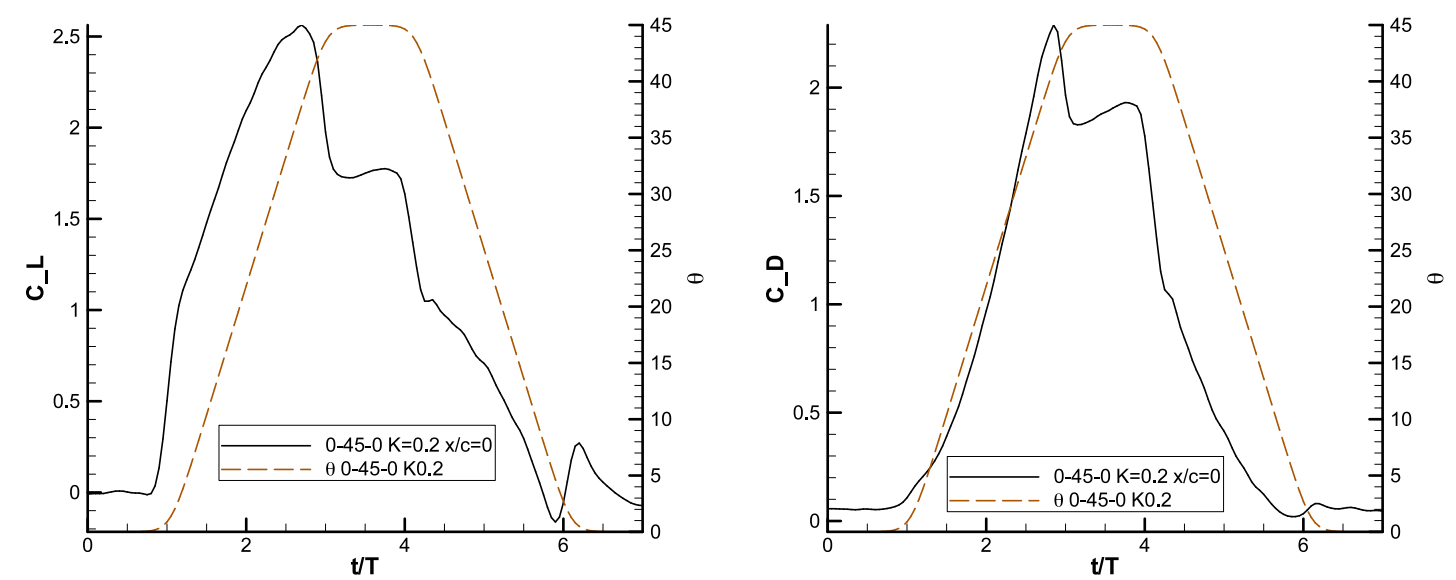

Figure 15. AIAA FDTC LRADG pitch ramp-hold-return, $0^{\circ}-45^{\circ}-0^{\circ}$, pivot at $\mathrm{x} / \mathrm{c}=0, \mathrm{~K}=0.2$.
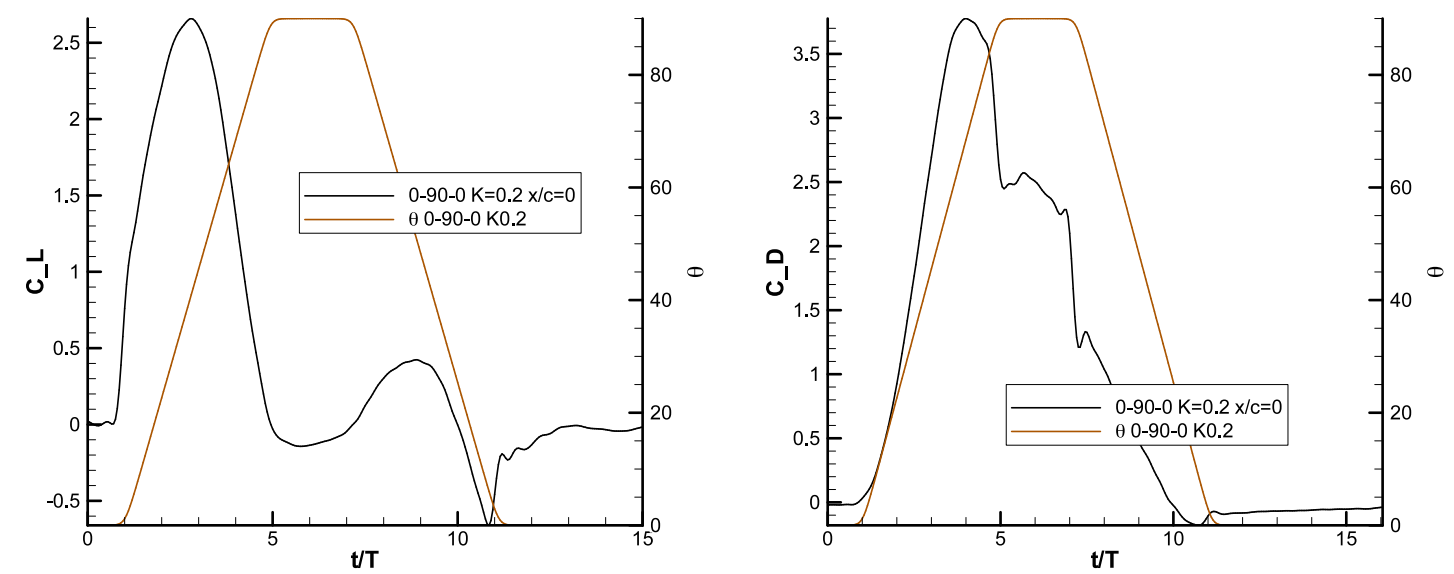

Figure 16. AIAA FDTC LRADG pitch ramp-hold-return, $0^{\circ}-90^{\circ}-0^{\circ}$, pivot at $\mathrm{x} / \mathrm{c}=\mathbf{0}, \mathrm{K}=0.2$.
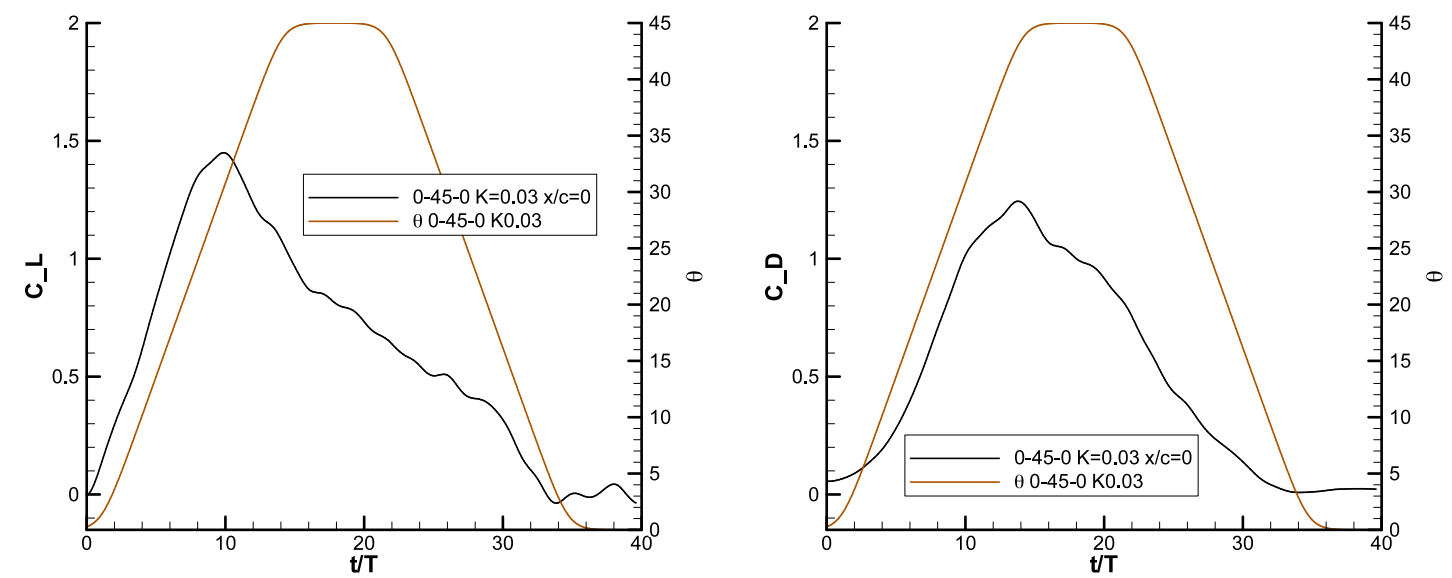

Figure 17. AIAA FDTC LRADG pitch ramp-hold-return, $0^{\circ}-45^{\circ}-0^{\circ}$, pivot at $\mathrm{x} / \mathrm{c}=\mathbf{0}, \mathrm{K}=\mathbf{0 . 0 3}$. 

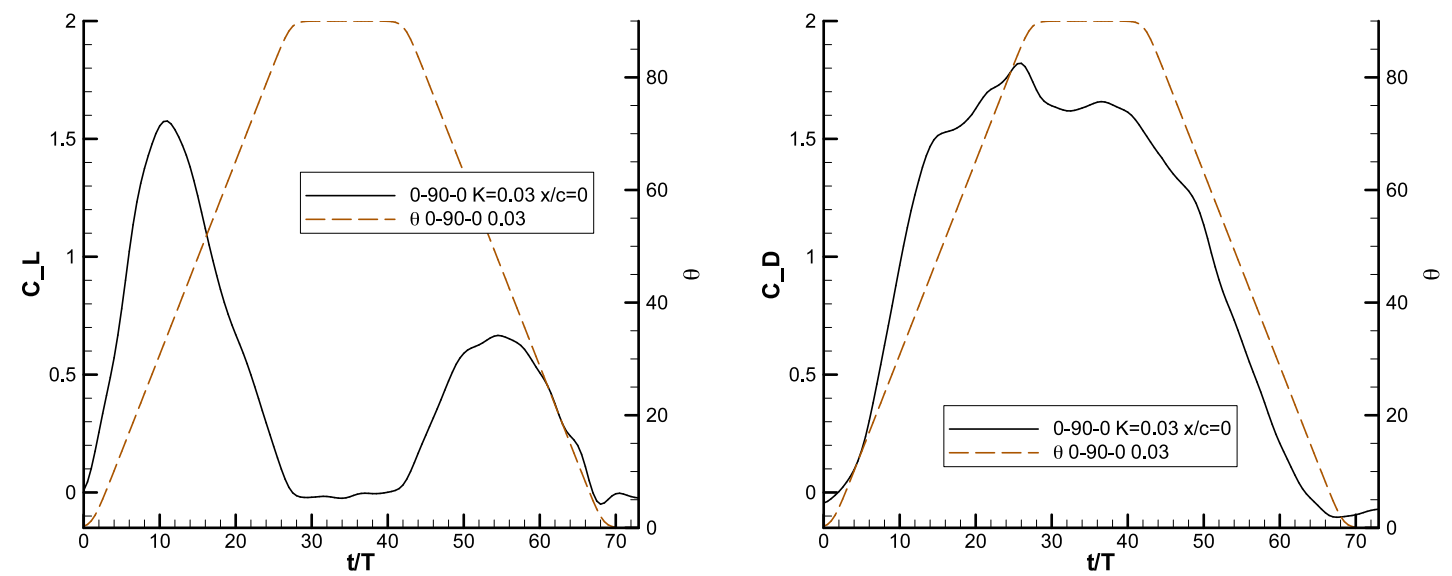

Figure 18. AIAA FDTC LRADG pitch ramp-hold-return, $0^{\circ}-90^{\circ}-0^{\circ}$, pivot at $\mathrm{x} / \mathrm{c}=\mathbf{0}, \mathrm{K}=0.03$.

Peak lift coefficient differs little between the 90-degree and 45-degree ramps, assuming the same reduced frequency. This suggests a saturation of lift at some angle below 45 degrees. For the 90-degree motion, the lift curve returns to zero when the plate reaches 90 degrees, with strong symmetry between the rising and falling portions of the lift curve on the upstroke.

\section{Correlations for Lift Coefficients}

Graham and Strickland ${ }^{2}$ proposed a relation for normalizing lift coefficient for a plate undergoing linear pitch ramp motion. First, one takes curves of $C_{L}$ vs. $\alpha$ for various $K$, extracts the maximum $\mathrm{C}_{\mathrm{L}}$ for each curve, and plots these values vs. $\mathrm{K}$. The least-squares linear fit to the $\mathrm{C}_{\mathrm{Lmax}} \mathrm{vs}$. $\mathrm{K}$ scatter plot is $m K+b$. Take $A \equiv m K+b$. Normalize the $\mathrm{C}_{\mathrm{L}}$ vs. $\alpha$ for various $\mathrm{K}$ by $A$, looking for the various lift curves to collapse onto one. Finally, Graham and Strickland rescale the oft-used theoretical relation, $C_{L}(\alpha)=2 A_{\text {lifi }} \sin \alpha \cos \alpha$, and assess favorably its fit to their measured lift data. Drag is treated similarly, with its own $A$-factor from the maximum $\mathrm{C}_{\mathrm{D}}$ for each $\mathrm{C}_{\mathrm{D}} \mathrm{vs}$. $\alpha$ curve, and $C_{D}(\alpha)=2 A_{\text {dras }} \sin ^{2} \alpha$. Linear fits to obtain $A_{\text {lift }}$ and $A_{\text {drag }}$ for the $2 \mathrm{D}$ plate and the $\mathrm{AR}=2$ rectangular plate undergoing 0-90 smoothed linear ramps are presented in Figure 19.
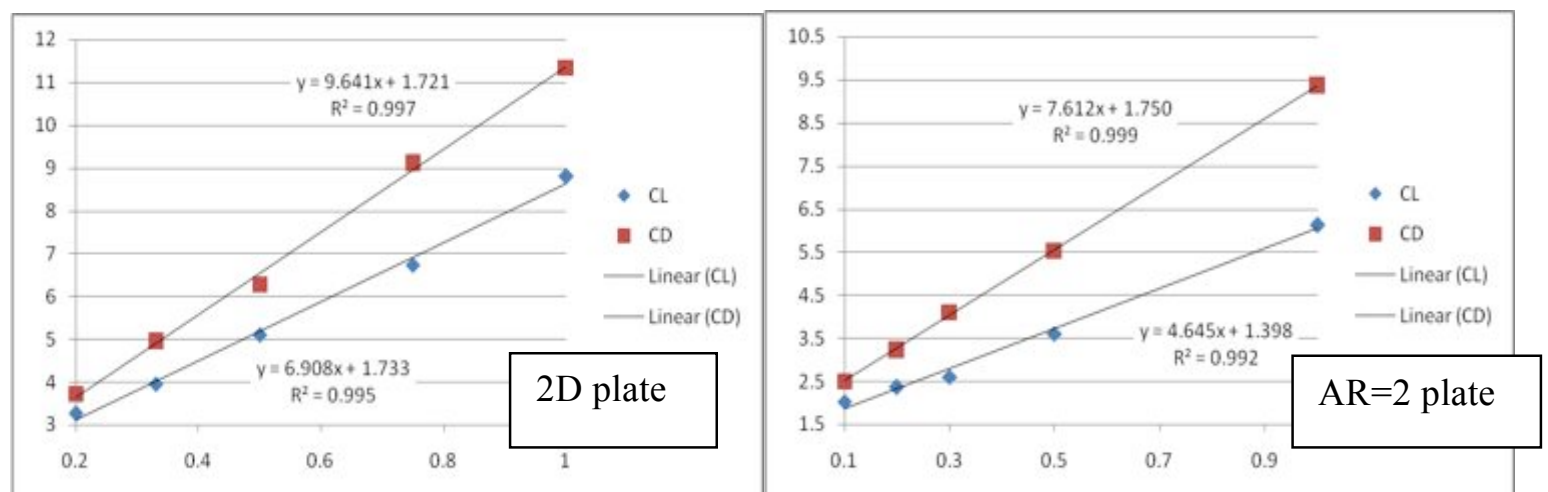

Figure 19. Linear fit to maximum lift and drag coefficients, after Graham and Strickland ${ }^{2}$, 0-90 smoothed linear pitch ramps; $2 D$ plate (left) and $A R=2$ rectangular plate (right), $K=0.2-1.0$. Leastsquares line fit defines $A_{\text {lift }}$ and $\boldsymbol{A}_{\text {drag }}$ for each respective plate. Pitch pivot point is x/c $=0.25$.

We pursue this relation for the $2 \mathrm{D}$ and $\mathrm{AR}=2$ rectangular plate motions, of the $0^{\circ}-90^{\circ}$ smoothed pitch ramps, at $\mathrm{K}=0.2$ through 1.0 (2D; Figure 20) and $\mathrm{K}=0.1$ through $1.0(\mathrm{AR}=2$; 
Figure 21). The only departure from Graham and Strickland is to ignore the noncirculatory force spikes, which for higher $\mathrm{K}$ values would otherwise overwhelm the circulatory $\mathrm{C}_{\mathrm{Lmax}}$.
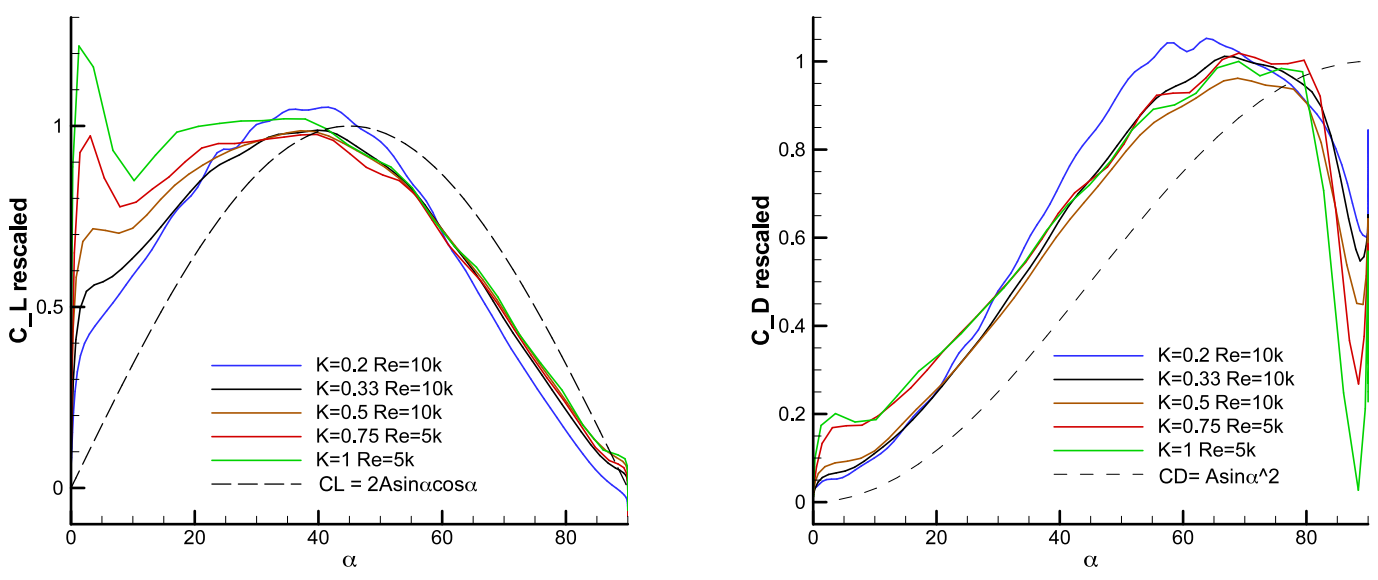

Figure 20. Rescaling of lift (lift) and drag (right) for 2D plate in 0-90 smoothed linear pitch ramp, after Graham and Strickland ${ }^{2}$; rescaled experimental data (solid lines) and theoretical curve fit (dashed lines).
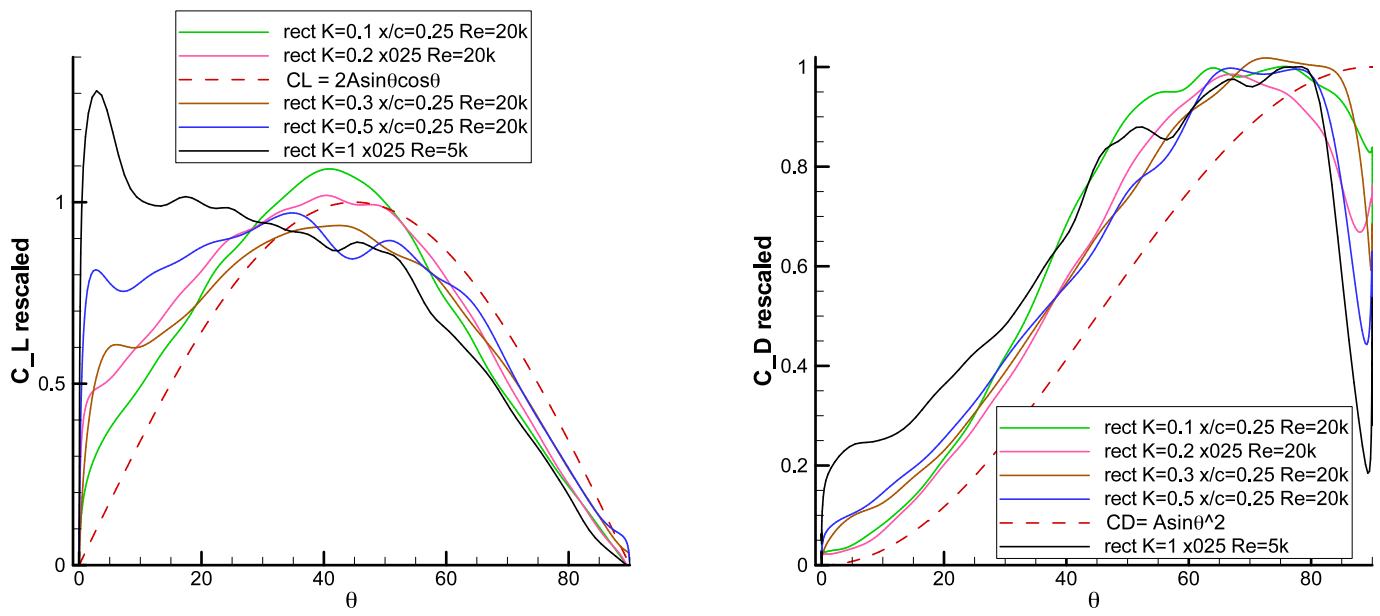

Figure 21. Rescaling of lift (lift) and drag (right) for $A R=2$ plate in 0-90 smoothed linear pitch ramp, after Graham and Strickland ${ }^{2}$; rescaled experimental data (solid lines) and theoretical curve fit (dashed lines).

The Graham-Strickland correlation is seen to be encouragingly successful at high angels of attack, even more so than in their original paper, perhaps owing to higher-resolution force measurements presently available. Again, this only holds provided that one ignores noncirculatory force. Scatter is nominally larger for the $\mathrm{AR}=2$ plate than for the $2 \mathrm{D}$ plate. For both plates, in lift the correlation fails at low angles of attack; below 20 degrees for the 2D plate, and below 30 degrees for the $\mathrm{AR}=2$ plate. The worst performance of the correlation is for the lowest reduced frequency. In drag, collapse of the various curves is fairly good at all angles of attack, with no notable exception for low angle of attack, where the noncirculatory contribution to drag is zero, or even at high angles of attack, where the rescaled noncirculatory spikes are rather similar to one another. Turning towards the theoretical inviscid quasi-steady fits for lift and drag, one finds much the same result as with the collapse of the various rescaled curves. In lift, agreement is good beyond the low angles of attack. In drag, the form of the theoretical and experiment curves is close, with the proviso that the experimental curves are shifted to the left - that is, to lower angles of attack. 
Rescaling by peak lift or drag coefficient destroys the lift curve slope comparison between the $2 \mathrm{D}$ and the $\mathrm{AR}=2$ plates. Lifting line theory and slender body theory both give a steady lift curve slope for the $\mathrm{AR}=2$ plate as half of that of the $2 \mathrm{D}$ plate. This relationship is roughly preserved in the correlations; the slope of the $A_{\text {lift }}$-factor for the 2D plate is 6.9 , while for the AR=2 plate it is 4.6. This is a factor of 1.5, as compared with the quasi-steady lift ratio of 2 .

The conceptual basis of the Graham-Strickland correlation is that peak aerodynamic force coefficients ought to scale linearly with $\mathrm{K}$. That the correlation can not apply to low reduced frequency is clear from the intercept in the $A$-factors not being zero. It is therefore appealing to attempt a direct proportionality. Brute trial and error suggests that lift, divided by reduced frequency to the $2 / 3$ rds power, results in a more or less successful rescaling for the $2 \mathrm{D}$ plate, but even here, the least successful collapse is for the lowest frequency, $\mathrm{K}=0.2$. For the $\mathrm{AR}=2$ plate, the best correlation is by using the $1 / 2$ power of reduced frequency. This ad hoc approach clearly begs for a broader parameter study of aspect ratio and reduced frequency, not to mention pivot point, if one is to aspire towards a universal scaling that phenomenologically accounts for the entire space of independent variables: pitch rate, aspect ratio, pitch pivot point location, and so forth.
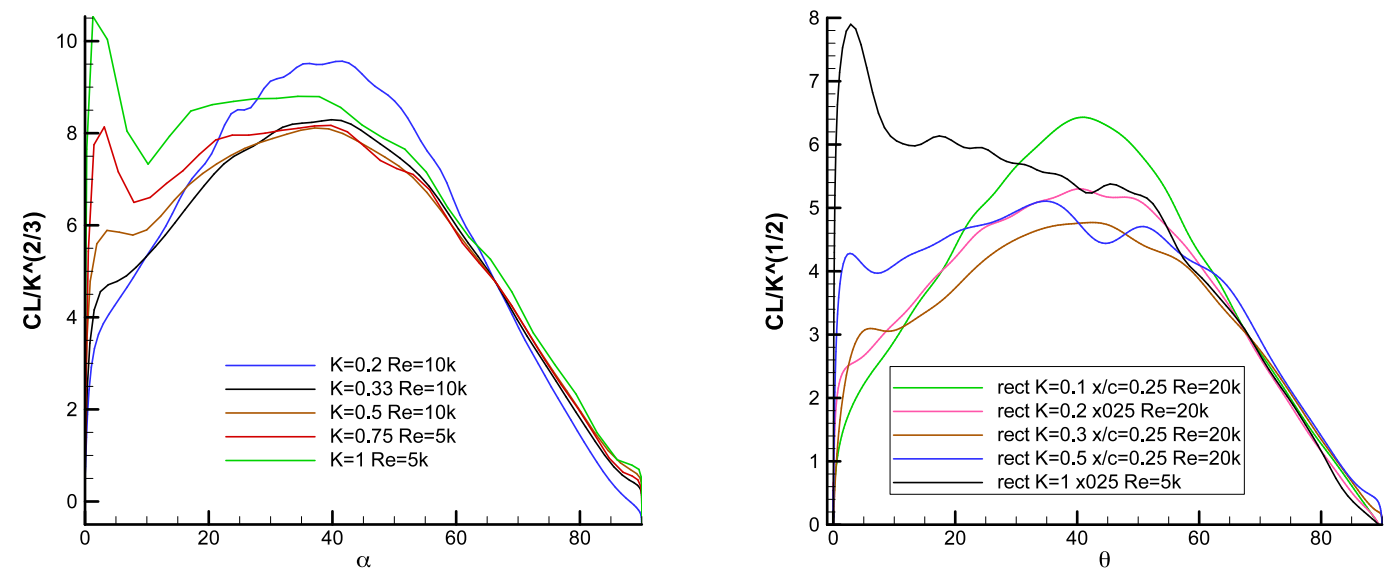

Figure 22. Ad hoc rescaling of lift coefficient in proportion to reduced frequency: 2D plate (left) and $A R=2$ plate (right), with exponent of reduced frequency as shown in the ordinate.

\section{Conclusion}

We examine a broad range of smoothed linear pitching motions, with reduced frequency from 0.003 through 2.0, focusing on 0.2-1.0. Two families of motions were considered: a pitch from 0 to 90 degrees incidence, and a pitch ramp-hold-return. Rectangular and Zimmerman plates of aspect ratio 2.0 were compared with a $2 \mathrm{D}$ plate. While the two $\mathrm{AR}=2$ plates evince considerable differences in leading edge vortex spanwise flow and tip-vortex/trailing vortex interaction, differences in their lift and drag histories are small. This suggests that it is not the off-body vortex system directly, but the bound circulation that determines most of the aerodynamic force history. For high reduced frequencies, peak lift no longer correlates with the saturation and shedding of the leading edge vortex, as this vortex takes time to form, and its formation can lag the motion kinematics and therefore the lift history severely. A classical rescaling of lift and drag coefficients, based on assumption of quasi-linear relation between maximum force coefficient and reduced frequency of pitch, was seen to be successful beyond that observed in the data set for which this scaling was developed, provided that one scales with respect to circulatory force, ignoring the noncirculatory lift spike at motion onset. 


\section{References}

${ }^{1}$ Dabiri, J.O. "Optimal Vortex Formation as a Unifying Principle in Biological Propulsion". Annual Rev. Fluid Mech., Vol. 41, pp. 17-33, 2009.

${ }^{2}$ Graham, G.M., and Strickland, J.H. "An Experimental Investigation of an Airfoil Pitching at Moderate to High Rates to Large Angles of Attack". AIAA 1986-0008.

${ }^{3}$ Granlund, K., OL, M., Garmann, D., Visbal, M., and Bernal, L. "Experiments and Computations on Abstractions of Perching". AIAA 2010-4943.

${ }^{4}$ Granlund, K., OL, M., and Bernal, L. "Experiments on Pitching Plates: Force and Flowfield Measurements at Low Reynolds Numbers". AIAA-2011-0872

${ }^{5}$ Eldredge, J.D., Toomey, J., and Medina, A. "On the Roles of Chord-Wise Flexibility in a Flapping Wing with Hovering Kinematics". Journal of Fluid Mechanics, Vol. 659, pp. 94-115, Sept. 2010.

${ }^{6}$ Koochesfahani, M.M., and Smiljanovski, V. "Initial Acceleration Effects on Flow Evolution around Airfoils Pitching to High Angle of Attack." AIAA Journal, Vol. 31, No. 8, pp. 1529-1531, 1993.

${ }^{7}$ Yilmaz, T., OL, M., and Rockwell, D. "Scaling of Flow Separation on a Pitching Low Aspect Ratio Plate". Journal of Fluids and Structures, Vol. 26, Issue 6, pp. 1034-1041, August 2010.

${ }^{8}$ Reich, G., Eastep, F., Altman, A., and Albertani, R. "Transient Post-Stall Aerodynamic Modeling for Extreme Maneuvers in Micro Air Vehicles". Journal of Aircraft, Vol. 48, No. 2, pp. 403-411, 2011.

${ }^{9}$ OL, M., Altman, A., Eldredge, J., Garmann, D., and Lian, Y. "Résumé of the AIAA FDTC Low Reynolds Number Discussion Group's Canonical Cases". AIAA-2010-1085. 